\title{
Counter-current chromatography for the separation of terpenoids: a comprehensive review with respect to the solvent systems employed
}

\author{
Krystyna Skalicka-Woźniak • Ian Garrard
}

Received: 15 January 2014/ Accepted: 11 March 2014/Published online: 25 March 2014

(C) The Author(s) 2014. This article is published with open access at Springerlink.com

\begin{abstract}
Natural products extracts are commonly highly complex mixtures of active compounds and consequently their purification becomes a particularly challenging task. The development of a purification protocol to extract a single active component from the many hundreds that are often present in the mixture is something that can take months or even years to achieve, thus it is important for the natural product chemist to have, at their disposal, a broad range of diverse purification techniques. Counter-current chromatography (CCC) is one such separation technique utilising two immiscible phases, one as the stationary phase (retained in a spinning coil by centrifugal forces) and the second as the mobile phase. The method benefits from a number of advantages when compared with the more traditional liquid-solid separation methods, such as no irreversible adsorption, total recovery of the injected sample, minimal tailing of peaks, low risk of sample denaturation, the ability to accept particulates, and a low solvent consumption.
\end{abstract}

K. Skalicka-Woźniak ( ()

Department of Pharmacognosy with Medicinal Plant Unit, Medical University of Lublin, 1 Chodzki Str., 20-093

Lublin, Poland

e-mail: kskalicka@pharmacognosy.org

I. Garrard

Advanced Bioprocessing Centre, Brunel Institute for Bioengineering, Brunel University, Uxbridge UB8 3PH, UK
The selection of an appropriate two-phase solvent system is critical to the running of CCC since this is both the mobile and the stationary phase of the system. However, this is also by far the most time consuming aspect of the technique and the one that most inhibits its general take-up. In recent years, numerous natural product purifications have been published using CCC from almost every country across the globe. Many of these papers are devoted to terpenoids-one of the most diverse groups. Naturally occurring terpenoids provide opportunities to discover new drugs but many of them are available at very low levels in nature and a huge number of them still remain unexplored. The collective knowledge on performing successful CCC separations of terpenoids has been gathered and reviewed by the authors, in order to create a comprehensive document that will be of great assistance in performing future purifications.

Keywords Counter-current chromatography ·

Terpenoids · Terpenes · Natural products .

Separation $\cdot$ Purification

\section{Introduction}

Terpenoids, also referred to as terpenes, are one of the largest and the most diverse group of natural products accounting for more than 40,000 individual compounds, with several new compounds being discovered every year. 


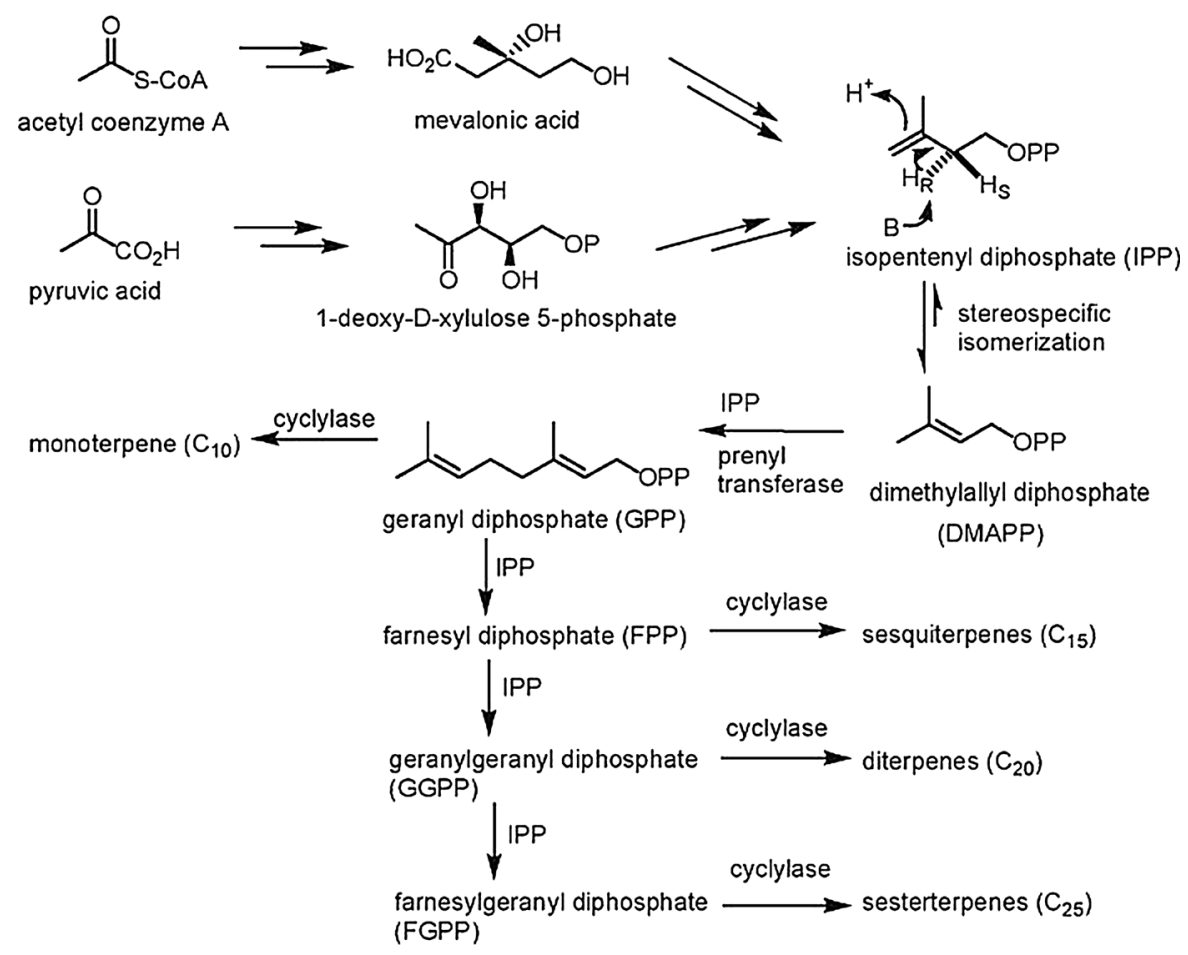

Fig. 1 Biosynthetic pathways of terpenes (Wang et al. 2005)

They are synthesized from only two five-carbon isomers: isopentenyl diphosphate (IPP) and dimethylallyl diphosphate (DMAPP). Two biosynthetic routes have been characterized: the classical acetate mevalonate pathway (described in 1967) and the triose phosphate-utilizing non-mevalonate pathway characterized in 2002. Starting from the universal precursors IPP and DMAPP, thousands of enzymes are involved in the biosynthetic pathways for terpenoid chain elongation, cyclization, and functionalization of hydrocarbon chains. The active isoprene unit (IPP) is repetitively added to DMAPP or a prenyl diphosphate in sequential head-to-tail condensations catalyzed by the prenyltrans-ferases. Through consecutive condensations a prenyltransferase can synthesize a variety of products with fixed lengths and stereochemistry (Fig. 1) (Wang et al. 2005; Ajikumar et al. 2008). Based on the number of the building blocks, terpenoids are commonly classified into hemi-, mono-, sesqui-, di-, ses-, tri- and tetraterpenoids (carotenoids) having $1,2,3,4,5,6$ and 8 isoprenoid residues respectively, and polyterpenes consisting of long chains of many isoprene units (Koch et al. 2008).

Terpenoids display a wide range of biological activities. Monoterpenes and sesquiterpenes are the main constituents of essential oils and share responsibility for important properties like antibacterial, antiviral, antioxidant etc. Triterpene saponins-ginsenosides-significantly reduce the production of beta-amyloid which accumulates in the brain of patients with Alzheimer's disease and play a critical role in pathology by inducing neuronal death. Ginkgolides (cyclic diterpenes of labdane type commonly isolated from Ginkgo biloba) protect neuronal cells from synaptic damage (Yoo and Park 2012). However the antimalarial drug Artemisininn and the anticancer drug paclitaxel (TaxolR) are two renowned terpenebased drugs with established medical applications. Artemisinin, earlier known as Qinghaosu, is a phytoconstituent isolated from Artemisia аппиа L. and can be described as a compound which possess antimalarial activity. Clinical studies with patients infected with Plasmodium vivax or P. falciparum demonstrated that artemisinin could kill the malarial parasite very quickly at the schizont stage of the parasite's life cycle (i.e. when it infects the human red blood cell) with no obvious side effects. The molecule has a completely new antimalarial prototype structure with an endoperoxide moiety, which is necessary for activity. Since artemisinin itself has poor bioavailability limiting its 
effectiveness, several semisynthetic derivatives of artemisinin have been developed (Medhi et al. 2009; Brown 2010). Taxol, a plant diterpenoid widely used as a chemotherapeutic drug against several types of cancer, is known to interact with a specific site of $\beta$ tubulin-it binds to microtubules and inhibits their disassembly. Cells treated with taxol are arrested in mitosis and eventually undergo death by apoptosis. This very important activity is strongly depended on its unusual structure. It was shown that the side chain at position $\mathrm{C}-13$ and the taxane ring system are essential for this activity (Xiang et al. 2009).

The purification of natural products is a complex process requiring a comprehensive range of techniques. CCC offers the natural product scientist a different mode of operation to conventional processes. Invented in the mid 1960's (Ito et al. 1966), to many scientists it is still known as it was back then-a technique that is slow, with separations measuring in hours or days. It was also unreliable as instruments frequently broke down and furthermore had poor capacity with injection amounts measured in tens of milligrams. There was also no opportunity for scale up as the factors required to scale up were poorly understood. However, the technique has been substantially developed since those early days. Advances in engineering and the understanding of the processes involved, particularly in the past 10 years, have created instruments that are fast, robust, permit very high injection loadings and, significantly for the natural products industry, can be rapidly scaled from analytical to pilot level (Sutherland and Fisher 2004). The new generation of coil planet centrifuges operate at higher " $g$ " fields than conventional instruments, enabling higher flow rates to be used so that separation times are measured in minutes rather than hours at the same resolution (Yuan et al. 2008). With a number of important advantages over both solid phase chromatographic techniques and current liquid-liquid extraction techniques, modern high capacity counter-current chromatography is a worthy inclusion in the array of techniques required for natural product purifications.

\section{Theoretical background of CCC}

In a counter-current chromatography centrifuge, tubing is wound on a drum which is centrifugally rotated in planetary motion (the holder rotates about its own

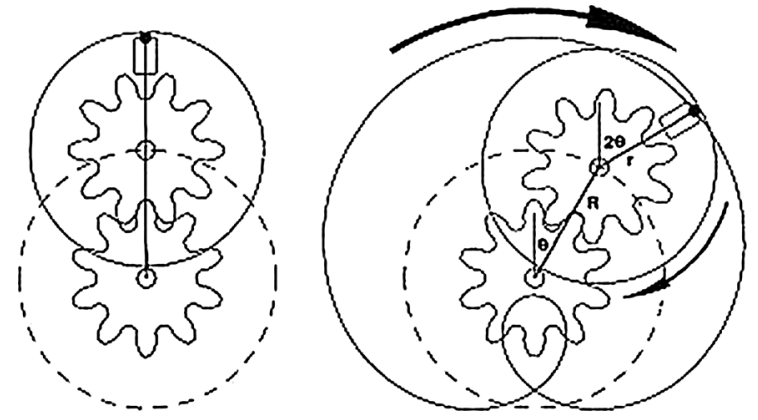

Fig. 2 Motion of the bobbin in the CCC centrifuge (Sutherland et al. 1998)

axis while revolving around the centrifuge axis at the same angular velocity in the same direction) (Fig. 2). A two phase solvent system is introduced into this coil. Although a simple solvent system might consist of hexane and water, a more likely system for a purification would consist of hexane, ethyl acetate, methanol and water or, for biomolecules sensitive to organic solvents, an aqueous two phase system such as aqueous PEG1000 and potassium phosphate salt solution. With the two phase solvent system inside, as the coil travels through its planetary motion cycle, zones of mixing and settling travel along the phases coincident with the low and high accelerations caused by the epicyclic motion of the coil. The mixing zones are coincident with low accelerations and take the form of wave mixing, equivalent to the "swishswosh" motion that occurs when a tube of liquids is tilted from side to side. The settling zones are coincident with high accelerations and take the form of a smooth interfacial area. There is one mixing and one settling zone per coil loop per revolution. A typical modern analytical CCC instrument may have 40 loops and spin at 2,000 rpm. It will therefore experience 4.8 million partitioning steps per hour $(40 \times 2,000 \times 60)$. Similarly, a typical preparative instrument may have 30 loops and spin at 1,200 rpm, giving 2.2 million partitioning steps per hour.

Counter-current chromatography can be achieved not only in the above mentioned hydrodynamic CCC created by two axis of rotation, but also as hydrostatic CCC, typically represented by centrifugal partition chromatography (CPC). This is a single-axis instrument, which has a series of chambers machined circumferentially around a rotor. Rotation of the rotor produces a uniform g-field, which retains the 


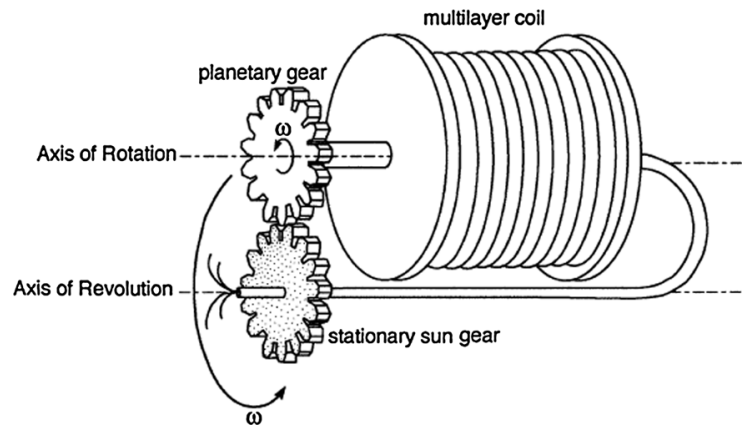

Fig. 3 Type-J planetary motion of a multilayer coil separation column presenting that the column holder rotates about its own axis and revolves around the centrifuge axis at the same angular velocity $(\omega)$ in the same direction (Ito 2005)

stationary phase in each chamber, while the mobile phase is flowed though in a cascading manner. A CPC instrument requires rotating seals for the mobile phase flow, whereas the hydrodynamic CCC instruments do not. This is because the rotation of the coil about its own axis unwinds the twist produced by its motion around the sun gear, and thus there is no twisting of the flow tubes linking the coil to the pump and the detector (Fig. 3).

\section{Solvent selection process}

In the past, selection of a suitable two phase solvent system involved a considerable amount of experience and know-how. With aqueous-organic phase systems coming from up to six or more different solvents mixed together, the possibilities were almost limitless. A typical mid-polarity selection table is shown in Table 1 (Garrard 2005). This is a modified version of the table produced by Oka et al. (1991) and runs from moderately polar (System No1: butanol-water) to moderately nonpolar (System No28: heptane-methanol). The italicized systems in the table can be used to rapidly screen the whole table first, allowing the operator to focus into the correct area of interest. Being all multiples of $0.5 \mathrm{ml}$ when $4 \mathrm{ml}$ solvent system is made, these particular systems are quick to make up and test. It is also possible to create the solvent systems on a micro-scale in 96 well plates if the crude sample is in short supply.

In order to achieve an efficient resolution of the target compounds, the $K$ values, which express the solute concentration in the stationary phase divided by
Table 1 Table for selecting a suitable moderately polar twophase solvent system, graded from polar (No1) to nonpolar (No28)

\begin{tabular}{|c|c|c|c|c|c|}
\hline No & Heptane & EtOAc & $\mathrm{MeOH}$ & Butanol & Water \\
\hline 1 & 0 & 0 & 0 & 2 & 2 \\
\hline 2 & 0 & 0.4 & 0 & 1.6 & 2 \\
\hline 3 & 0 & 0.8 & 0 & 1.2 & 2 \\
\hline 4 & 0 & 1.2 & 0 & 0.8 & 2 \\
\hline 5 & 0 & 1.6 & 0 & 0.4 & 2 \\
\hline 6 & 0 & 2 & 0 & 0 & 2 \\
\hline 7 & 0.1 & 1.9 & 0.1 & 0 & 1.9 \\
\hline 8 & 0.2 & 1.8 & 0.2 & 0 & 1.8 \\
\hline 9 & 0.29 & 1.71 & 0.29 & 0 & 1.71 \\
\hline 10 & 0.33 & 1.67 & 0.33 & 0 & 1.67 \\
\hline 11 & 0.4 & 1.6 & 0.4 & 0 & 1.6 \\
\hline 12 & 0.5 & 1.5 & 0.5 & 0 & 1.5 \\
\hline 13 & 0.57 & 1.43 & 0.57 & 0 & 1.43 \\
\hline 14 & 0.67 & 1.33 & 0.67 & 0 & 1.33 \\
\hline 15 & 0.8 & 1.2 & 0.8 & 0 & 1.2 \\
\hline 16 & 0.91 & 1.09 & 0.91 & 0 & 1.09 \\
\hline 17 & 1 & 1 & 1 & 0 & 1 \\
\hline 18 & 1.09 & 0.91 & 1.09 & 0 & 0.91 \\
\hline 19 & 1.2 & 0.8 & 1.2 & 0 & 0.8 \\
\hline 20 & 1.33 & 0.67 & 1.33 & 0 & 0.67 \\
\hline 21 & 1.43 & 0.57 & 1.43 & 0 & 0.57 \\
\hline 22 & 1.5 & 0.5 & 1.5 & 0 & 0.5 \\
\hline 23 & 1.6 & 0.4 & 1.6 & 0 & 0.4 \\
\hline 24 & 1.67 & 0.33 & 1.67 & 0 & 0.33 \\
\hline 25 & 1.71 & 0.29 & 1.71 & 0 & 0.29 \\
\hline 26 & 1.8 & 0.2 & 1.8 & 0 & 0.2 \\
\hline 27 & 1.9 & 0.1 & 1.9 & 0 & 0.1 \\
\hline 28 & 2 & 0 & 2 & 0 & 0 \\
\hline
\end{tabular}

Quantities (in $\mathrm{ml}$ ) required to make $4 \mathrm{ml}$ of system using a liquid-handling robot. EtOAc = ethyl acetate, $\mathrm{MeOH}=$ methanol. Hexane may be used instead of heptane (Garrard 2005)

that in the mobile phase, should be calculated. The partition coefficient $(K)$ should lie within the approximate range $0.5<K<2.0$. A smaller $K$ value results in a loss of peak resolution, whilst a larger value produces excessive band broadening.

\section{Solvent systems employed in CCC for terpenoid purification}

In order to fully assess the use of two phase systems in CCC of terpenoids, approximately 3,500 scientific 
papers were studied, published in the last 30 years, that related to all aspects of CCC or CPC. Papers that contained an application example of terpenoids, i.e. a purification performed by CCC or CPC were noted, together with the compounds purified and the solvent system used for the purification. Papers which gave examples of separations reported elsewhere were ignored, as were all symposium abstracts. Only papers which gave specific details of the solvent system and solute were recorded and only natural product secondary metabolites were noted e.g. no synthetic compounds, dyes or chemicals.

In total therefore, 150 solvent systems were listed in Table 2 together with the corresponding solutes that they separated. Some of the solvent systems corresponded to more than one solute, and some of the solutes corresponded to more than one solvent system, but if the same solute and the same solvent system were listed, this was simply a duplicate entry and was therefore removed.

The solvent systems tables mentioned above are presented here, sorted according to class of terpenoid separated. That table is presented for the benefit of CCC users by suggesting possible suitable solvent systems, which can act as a starting point for further refinement and optimization in the composition of the system.

\section{Applications of high speed and high performance CCC}

Being a liquid-liquid chromatography system, CCC can select from an almost infinite range of possible two-phase solvent systems for a purification. Most reported purifications with the technique have understandably concentrated on compounds of intermediate polarity. For example, a review of Chinese herbal medicines purified by CCC found a total of 214 different compounds in 198 published papers with a LogP polarity range from -4 to +12 (Sutherland and Fisher 2009). However, more than $60 \%$ of those compounds fell in the narrow intermediate polarity range of 0-4. Nevertheless, CCC and its sister technique, CPC can be particularly useful for purifications in the extreme polar and non-polar range and some impressive examples have been published. In 1995, Gasper and co-workers managed to purify $\mathrm{C}_{60}$ and $\mathrm{C}_{70}$ fullerenes using the non-polar and non- aqueous solvent system of isooctane, dimethylformamide, 1,2-dichlorobenzene $(4: 2: 1)$ plus $1 \%$ tertbutylmethyl ether (Gasper et al. 1995). Still at the nonpolar end of the spectrum, the carotenoid lycopene was isolated from tomato paste using a non-aqueous phase system of $n$-hexane, dichloromethane and acetonitrile (10:3.5:6.5) (Wei et al. 2001). This separation was performed in a single step from the crude material with $100 \mathrm{mg}$ of crude extract injected onto a $230 \mathrm{ml}$ capacity centrifuge. The purity of the final product was measured as $>98 \%$ by HPLC peak area. Lycopene has a calculated $\log \mathrm{P}$ value of approximately 17.6. Beyond the carotenoids, nonpolar terpenoids can also be challenging to purify with solid phase chromatography, and again CCC offers an alternative approach. In one example, Liu et al. (2013a) successfully separated cycloartenyl ferulate and 24-methylene cycloartanyl ferulate. Due to the weak polarity, a series of low-polar solvent systems, including: $n$-hexane-ethyl acetate-ethanol (methanol)-water, $n$-hexane-ethanol (methanol)-water, $n$ hexane-methanol and $n$-hexane-ethyl acetate- $n$-butanol ethanol (methanol)-water were tested, but all without success. Since the HPLC mobile phase for the analysis of those two target compounds consisted of methanol, acetonitrile and isopropanol, these three solvents were further studied and a mixture of hexane and acetonitrile (1:1) was used for the purification. Another application of non-aqueous solvent systems is the purification of shionone-also a low-polar compound. Wang et al. (2012a) tested several hydrophobic two-phase solvent systems. Among these $n$-hexanemethanol (2:1) and heptane-dichloromethane-acetonitrile (20:7:13) were suitable for the separation.

Turning to the extreme polar end of the spectrum, CCC has been used with aqueous-organic solvent systems for the purification of peptides. For example, the peptide antibiotic colistin ( $\log \mathrm{P}$ about -4.7$)$ was isolated from a commercial microbial preparation using the polar two-phase system consisting of $n$ butanol and $0.04 \mathrm{M}$ aqueous trifluoroacetic acid (1:1) (Ikai et al. 1998). Using the salt-based solvent system consisting of 1-propanol, acetonitrile, saturated ammonium sulphate and water (1:0.5:1.2:1) the highly polar glucosinolate glucoraphanin was purified to a purity $>98 \%$ from a crude broccoli extract in a single step (Fisher et al. 2005).

Sometimes modifications are necessary. The separation of three closely related triterpenes: sericic acid, 
Table 2 Solvent systems in CCC for terpenoids separations

\begin{tabular}{|c|c|c|c|}
\hline $\begin{array}{l}\text { Classes of } \\
\text { compounds }\end{array}$ & Purified compounds & References & Type of apparatus/solvent system \\
\hline \multirow[t]{12}{*}{ Monoterpenoids } & $\begin{array}{l}\text { Paeoniflorin } \\
\text { Albiflorin }\end{array}$ & Huang et al. (2013) & $\begin{array}{l}\text { CCC/ethyl acetate- } n \text {-butanol- } \\
\text { water }(3: 2.5: 5)\end{array}$ \\
\hline & $\begin{array}{l}\text { Rosiridin } \\
\text { Geranyl 1- } O \text { - } \alpha \text {-L-arabinopyranosyl- } \\
\quad(1 \rightarrow 6) \text { - } \beta \text {-D-glucopyranoside }\end{array}$ & Mudge et al. (2013) & $\begin{array}{l}\text { CCC/ethyl acetate-butanol-water } \\
(3: 2: 5)\end{array}$ \\
\hline & $(S)$-3,7-Dimethyl-5-octene-1,7-diol & Knapp et al. (1998) & $\begin{array}{l}\text { CCC/chloroform-methanol-water } \\
(7: 13: 8)\end{array}$ \\
\hline & $\begin{array}{l}\text { Thymol } \\
\text { Carvacrol }\end{array}$ & $\begin{array}{l}\text { Puertas Mejia et al. } \\
\text { (2002) }\end{array}$ & $\begin{array}{l}\mathrm{CCC} / n \text {-hexane-tert-butylmethyl } \\
\text { ether-acetonitrile }(1: 0.1: 1)\end{array}$ \\
\hline & Eugenol & Geng et al. (2007) & $\begin{array}{l}\text { CCC/n-hexane-ethyl acetate- } \\
\text { methanol-water }(1: 0.5: 1: 0.5)\end{array}$ \\
\hline & $\begin{array}{l}\text { Chavibetol } \\
\text { Methyleugenol }\end{array}$ & dos Santos et al. (2009) & $\begin{array}{l}\text { CCC/n-hexane-n-butanol- } \\
\text { methanol-water }(12: 4: 4: 3)\end{array}$ \\
\hline & $\alpha$-Cyperone & Shi et al. (2009) & $\begin{array}{l}\mathrm{CCC} / n \text {-hexane-ethyl acetate- } \\
\text { methanol-water }(1: 0.2: 1.1: 0.2)\end{array}$ \\
\hline & 1,8-Cineole & Dang et al. (2010) & $\begin{array}{l}\mathrm{CPC} / \text { petroleum ether-acetonitrile- } \\
\text { acetone }(4: 3: 1)\end{array}$ \\
\hline & Paeoniflorin & Chen et al. (2004) & $\begin{array}{l}\text { CCC/n-butanol-ethyl acetate- } \\
\text { water }(1: 4: 5)\end{array}$ \\
\hline & $\begin{array}{l}\text { Cuminaldehyde } \\
p \text {-Menta-1,4-dien-7-al }\end{array}$ & Chen et al. (2011) & $\begin{array}{l}\text { CCC/n-hexane-methanol-water } \\
(5: 4: 1)\end{array}$ \\
\hline & $\begin{array}{l}\text { Linalol } \\
\text { Terpinen-4-ol } \\
\alpha \text {-Terpineol }\end{array}$ & $\begin{array}{l}\text { Skalicka-Woźniak et al. } \\
\text { (2013) }\end{array}$ & $\begin{array}{l}\text { CCC/heptane-ethyl acetate- } \\
\text { methanol-water }(5: 2: 5: 2)\end{array}$ \\
\hline & $\begin{array}{l}\text { Anethole } \\
\text { Foeniculin }\end{array}$ & $\begin{array}{l}\text { Skalicka-Woźniak et al. } \\
\text { (2013) }\end{array}$ & CCC/heptane-methanol (1:1) \\
\hline \multirow[t]{4}{*}{ Sesquiterpenoids } & 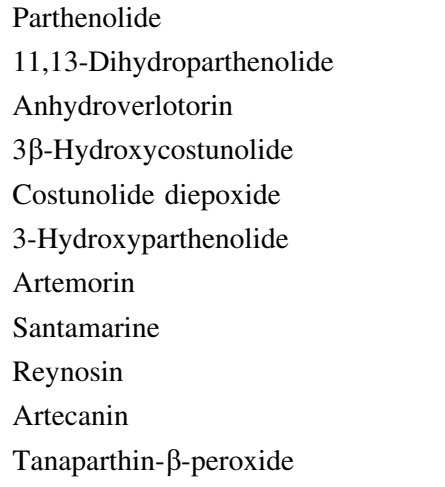 & Fischedick et al. (2012) & $\begin{array}{l}\text { CPC/heptane-ethyl acetate- } \\
\text { methanol-water }(1: 1: 1: 1)\end{array}$ \\
\hline & Artemisinin & Acton et al. (1986) & $\begin{array}{l}\text { CCC/iso-octane-ethyl acetate- } \\
\text { methanol-water }(7: 3: 6: 4)\end{array}$ \\
\hline & $\begin{array}{l}\text { Costunolide } \\
\text { Dehydrocostuslactone }\end{array}$ & Li et al. (2005) & $\begin{array}{l}\text { CCC/light petroleum-methanol- } \\
\text { water }(5: 6.5: 3.5)\end{array}$ \\
\hline & Lactucopicrin & Wu et al. (2007) & $\begin{array}{l}\text { CCC/n-hexane-ethyl acetate- } \\
\text { methanol-water }(1.5: 5: 2.75: 5)\end{array}$ \\
\hline
\end{tabular}


Table 2 continued

\begin{tabular}{|c|c|c|c|}
\hline $\begin{array}{l}\text { Classes of } \\
\text { compounds }\end{array}$ & Purified compounds & References & Type of apparatus/solvent system \\
\hline & $11 \beta, 13$-Dihydrolactucin & \multirow[t]{2}{*}{ Wu et al. (2007) } & \multirow[t]{2}{*}{ CCC/ethyl acetate-methanol-water (20:1:20) } \\
\hline & Lactucin & & \\
\hline & Peroxyferolide & \multirow[t]{2}{*}{ Graziose et al. (2011) } & \multirow[t]{2}{*}{ CPC/n-hexane-ethyl acetate-methanol-water $(2: 1: 2: 1)$} \\
\hline & Lipiferolide & & \\
\hline & $\begin{array}{l}\text { 14-(3-Methylpentanoyl)-6- } \\
\text { deoxybritannilactone }\end{array}$ & \multirow[t]{10}{*}{ Fischedick et al. (2013a) } & \multirow[t]{10}{*}{ CPC/heptane-ethyl acetate-methanol-water (4:6:4:6) } \\
\hline & $\begin{array}{l}\text { 14-(3-Methylbutanoyl)-6- } \\
\text { deoxybritannilactone }\end{array}$ & & \\
\hline & $\begin{array}{l}\text { 14-(2-Methylpropanoyl)-6- } \\
\text { deoxybritannilactone }\end{array}$ & & \\
\hline & 1,3-Epi-granilin & & \\
\hline & $\begin{array}{l}\text { 11,13-Dihydro- } \\
\text { inuchinenolide B }\end{array}$ & & \\
\hline & Pulchellin C & & \\
\hline & 6-Deacetylbritanin & & \\
\hline & 4H-Tomentosin & & \\
\hline & Gaillardin & & \\
\hline & Britannin & & \\
\hline & $\begin{array}{l}3 \beta \text {-Hydroxy- } 8 \beta-\left[4^{\prime}-\right. \\
\text { hydroxytigloyloxy]- } \\
\text { costunolide }\end{array}$ & \multirow[t]{3}{*}{ Yan et al. (2012) } & \multirow[t]{3}{*}{$\mathrm{CCC} / n$-hexane-ethyl acetate-methanol-water $(1: 4: 2: 3)$} \\
\hline & Eupalinolide A & & \\
\hline & Eupalinolide B & & \\
\hline & Xanthathin & \multirow[t]{3}{*}{ Pinel et al. (2007) } & \multirow[t]{3}{*}{$\mathrm{CPC} / n$-hexane-ethyl acetate-methanol-water $(1: 1: 1: 1)$} \\
\hline & 4-Epi-xanthanol & & \\
\hline & 4-Epi-isoxanthanol & & \\
\hline & $\beta$-Caryophyllene & Xie et al. (2008) & $\mathrm{CCC} / n$-hexane-dichloromethane-acetonitrile (10:3:7) \\
\hline & Rupestonic acid & Ma et al. (2005) & $\begin{array}{l}\text { CCC/n-hexane-ethyl acetate-methanol-water } \\
(6: 4: 3.5: 6.5) \text { with } 0.5 \% \text { acetic acid in stationary-phase }\end{array}$ \\
\hline & Rupestonic acid & Yang et al. (2010) & CCC/n-hexane-ethyl acetate-methanol-water $(3: 5: 3: 5)$ \\
\hline & Germacrone & Yan et al. (2005) & CCC/light petroleum ether-ethanol-diethyl ether-water \\
\hline & Curdione & & $(5: 4: 0.5: 1)$ \\
\hline & Atractylon & \multirow[t]{2}{*}{ Zhao and $\mathrm{He}(2006)$} & \multirow{2}{*}{$\begin{array}{l}\text { CCC/light petroleum-ethyl acetate-ethanol-water } \\
(4: 1: 4: 1)\end{array}$} \\
\hline & Atractylenolide III & & \\
\hline & Nootkatone & Xie et al. (2009) & CCC/n-hexane-methanol-water $(5: 4: 1)$ \\
\hline & Caryophyllene oxide & \multirow[t]{3}{*}{ Wei et al. (2012) } & \multirow[t]{3}{*}{$\mathrm{CCC} / n$-hexane-acetonitrile-ethanol $(5: 4: 3)$} \\
\hline & $\beta$-Farnesene & & \\
\hline & Caryophyllene & & \\
\hline & $(S)$-Dehydrovomifoliol & Yang et al. (2013) & CCC/n-hexane-ethyl acetate-methanol-water $(1: 5: 1: 5)$ \\
\hline & Curdione & \multirow[t]{5}{*}{ Dang et al. (2010) } & \multirow[t]{5}{*}{ CPC/light petroleum ether-acetonitrile-acetone $(4: 3: 1)$} \\
\hline & Curcumol & & \\
\hline & Germacrone & & \\
\hline & Curzerene & & \\
\hline & $\beta$-Elemene & & \\
\hline & Patchoulol & Li et al. (2011) & CPC/light petroleum ether-acetonitrile $(1: 1)$ \\
\hline
\end{tabular}


Table 2 continued

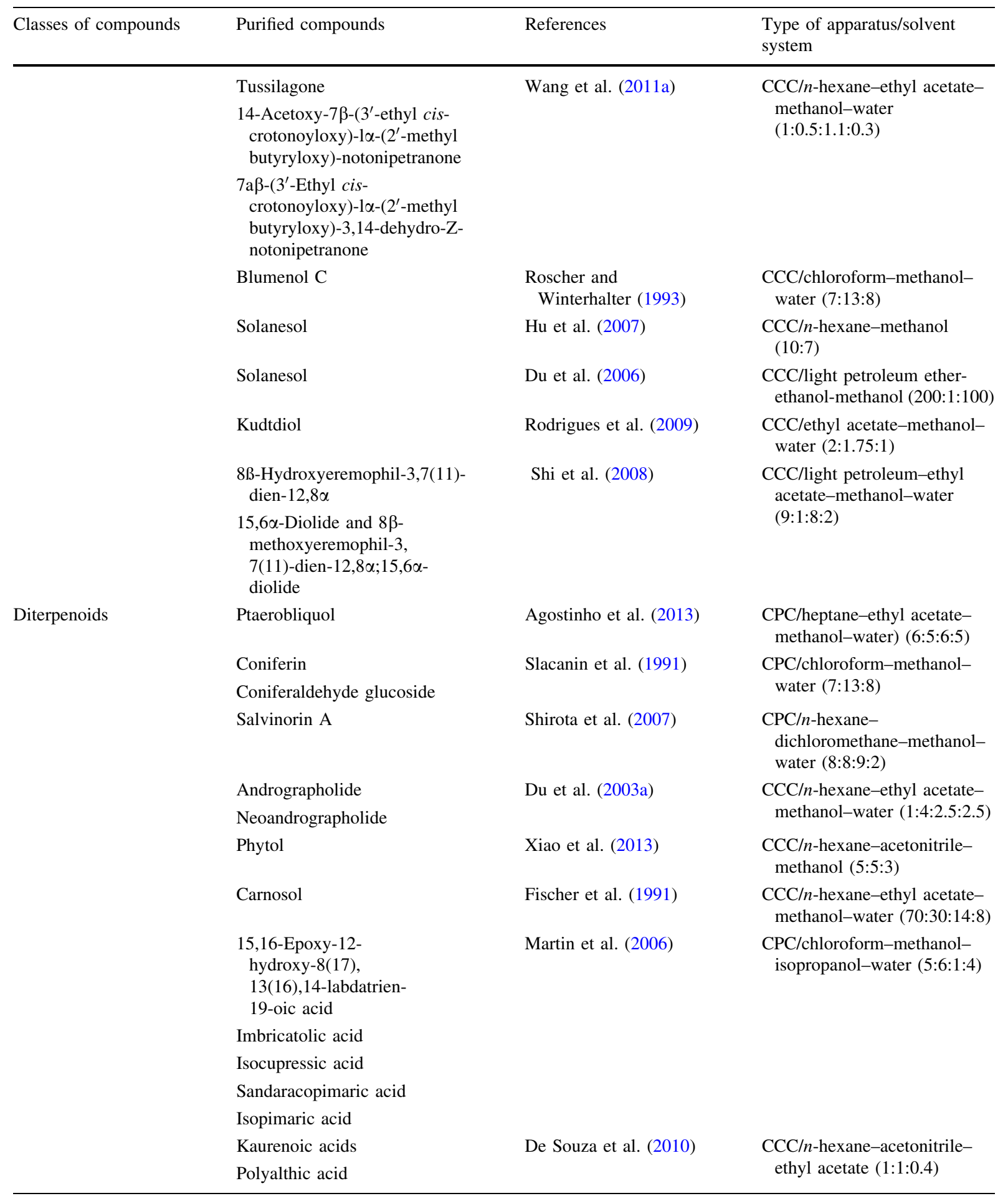


Table 2 continued

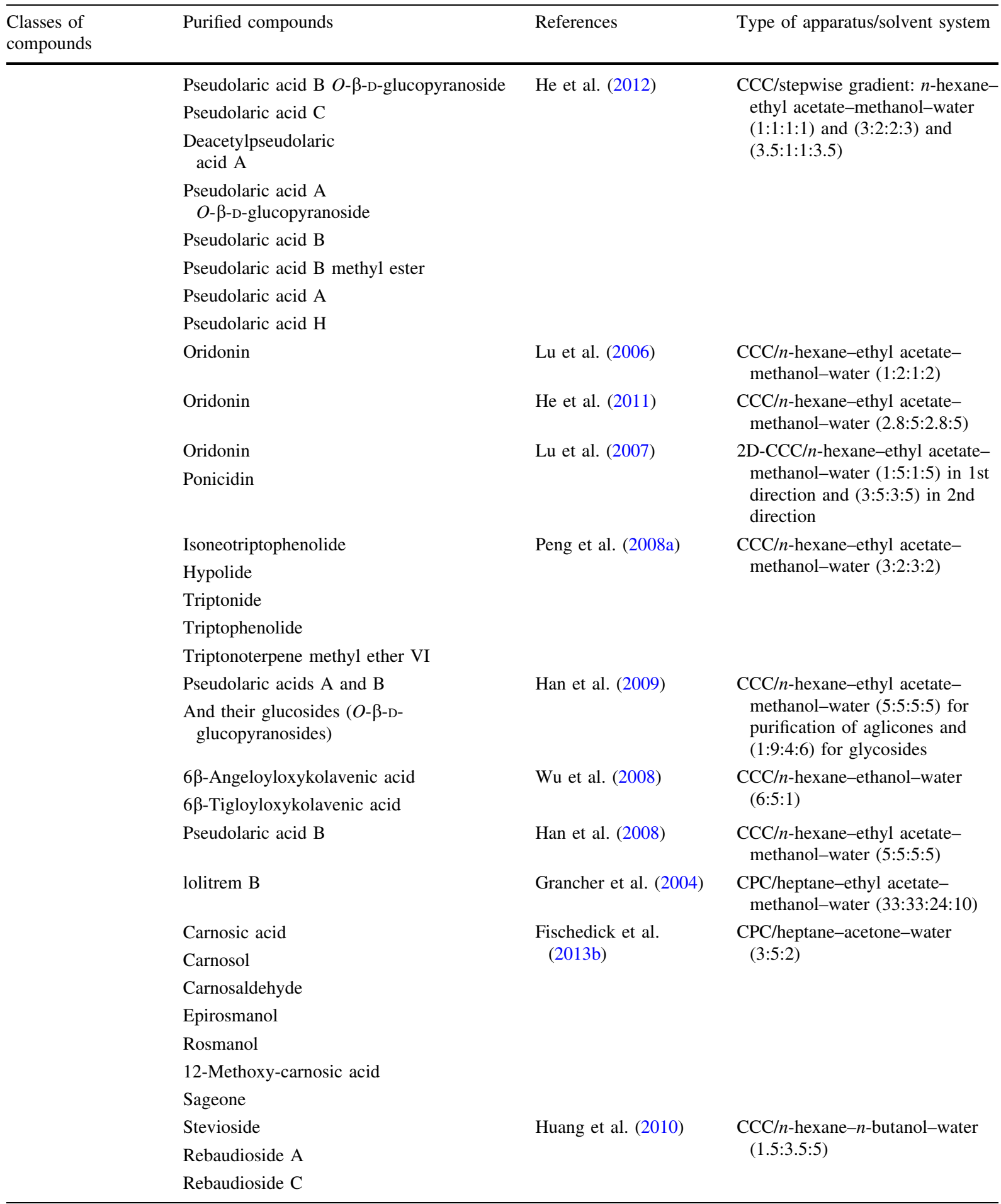


Table 2 continued

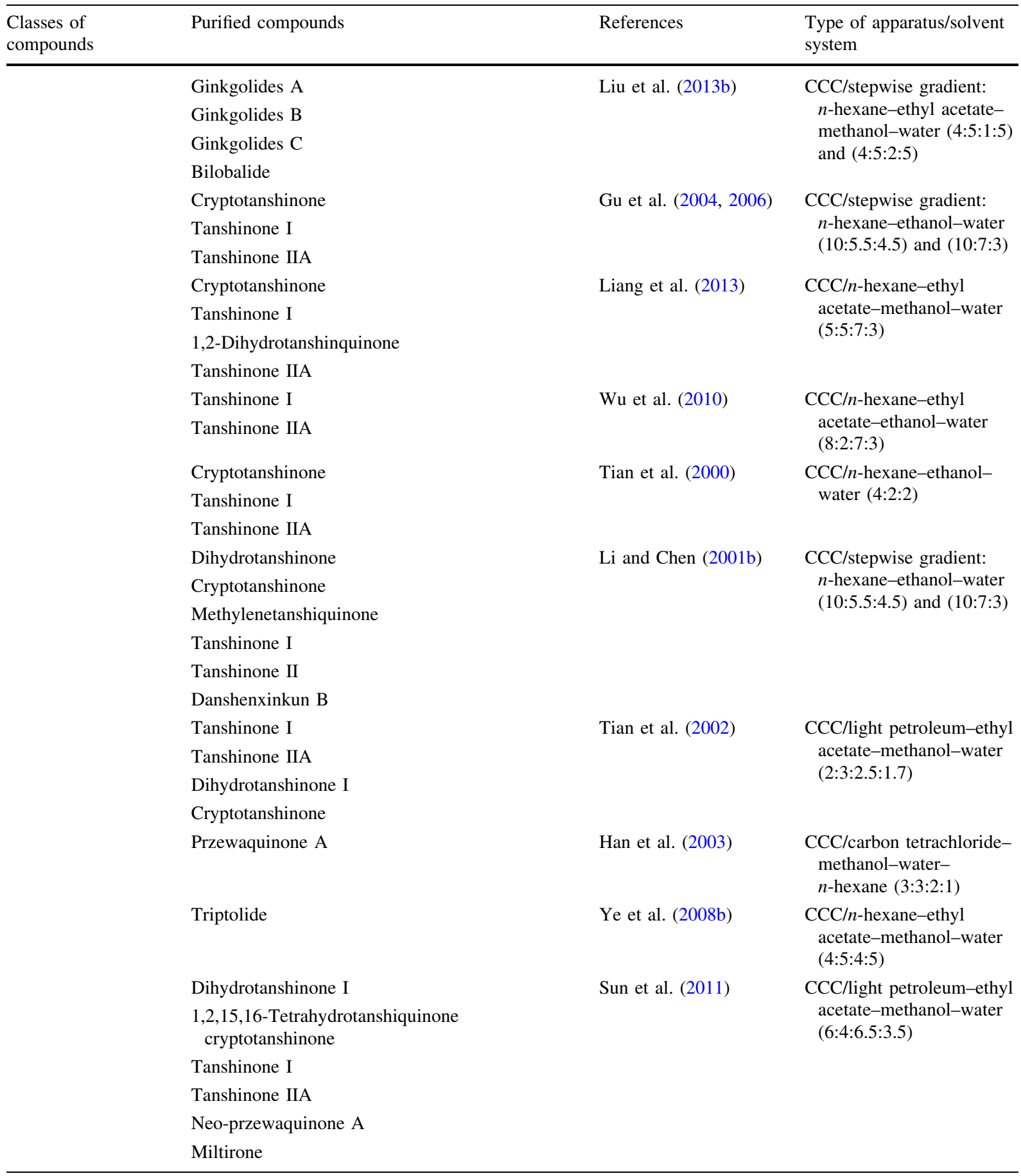


Table 2 continued

\begin{tabular}{|c|c|c|c|}
\hline Classes of compounds & Purified compounds & References & Type of apparatus/solvent system \\
\hline \multirow[t]{31}{*}{ Triterpenoids } & $\begin{array}{l}2 \beta, 3 \beta, 4 \beta \text {-Trihydroxypregnan-16- } \\
\text { one }\end{array}$ & Rodrigues et al. (2009) & $\begin{array}{l}\mathrm{CCC} / n \text {-hexane-ethyl acetate-methanol- } \\
\text { water }(1: 2: 1.75: 1)\end{array}$ \\
\hline & Squalene & Lu et al. (2003) & $\mathrm{CCC} / n$-hexane-methanol $(2: 1)$ \\
\hline & $\begin{array}{l}\text { Pristimerin } \\
\text { Netzahualcoyene }\end{array}$ & Gutiérrez et al. (2007) & $\begin{array}{l}\text { CPC/heptane-ethyl acetate-methanol- } \\
\text { water }(8: 1: 6: 1)\end{array}$ \\
\hline & Guyanin & Severino et al. (2009) & $\begin{array}{l}\mathrm{CCC} / n \text {-hexane-ethanol-acetonitrile- } \\
\text { water }(10: 8: 1: 1)\end{array}$ \\
\hline & Barbinervic acid & \multirow[t]{4}{*}{ Fan and $\mathrm{He}(2006)$} & \multirow{4}{*}{$\begin{array}{l}\mathrm{CCC} / n \text {-hexane-ethyl acetate-methanol- } \\
\text { water }(3: 6: 4: 2)\end{array}$} \\
\hline & Rotungenic acid & & \\
\hline & 24-Hydroxy ursolic acid & & \\
\hline & Ursolic acid & & \\
\hline & Betulinic acid & Frighetto et al. (2005) & $\begin{array}{l}\mathrm{CCC} / n \text {-hexane-ethyl acetate-methanol- } \\
\text { water }(10: 5: 2.5: 1)\end{array}$ \\
\hline & Ursolic acid & Frighetto et al. (2008) & $\begin{array}{l}\mathrm{CCC} / n \text {-hexane-ethyl acetate-methanol- } \\
\text { water }(10: 5: 2.5: 1)\end{array}$ \\
\hline & Bellericagenin B & \multirow[t]{4}{*}{ Nasser et al. (2006) } & \multirow{4}{*}{$\begin{array}{l}\text { CCC/chloroform-methanol-water } \\
(43: 37: 20)\end{array}$} \\
\hline & Bellericaside B & & \\
\hline & Arjunglucoside I & & \\
\hline & $\begin{array}{r}\text { 28-Nor-17, 22-seco-2 } \alpha, 3 \beta, 19,22, \\
23 \text {-pentahydroxy- } \Delta \text { 12-Oleanane }\end{array}$ & & \\
\hline & $\begin{array}{l}2 \alpha, 3 \alpha, 19 \beta, 23 \beta \text {-Tetrahydroxyurs- } \\
12 \text {-en-28-oic acid }\end{array}$ & \multirow[t]{2}{*}{ Liu et al. (2011) } & \multirow[t]{2}{*}{$\begin{array}{l}\mathrm{CCC} / n \text {-hexane-ethyl acetate-methanol- } \\
\text { water }(10: 5: 3: 1)\end{array}$} \\
\hline & $\begin{array}{l}2 \alpha, 3 \alpha, 23 \beta \text {-Trihydroxyurs-12-en- } \\
28 \text {-oic acid }\end{array}$ & & \\
\hline & Oleanolic acid & \multirow[t]{2}{*}{ Du et al. (1995) } & \multirow{2}{*}{$\begin{array}{l}\mathrm{CCC} / n \text {-hexane-ethyl acetate-methanol- } \\
\text { water }(3: 6: 2: 1)\end{array}$} \\
\hline & Ursolic acid & & \\
\hline & Shionone & Wang et al. (2012a) & $\mathrm{CCC} / n$-hexane-methanol $(2: 1)$ \\
\hline & Taraxasterol acetate & \multirow[t]{3}{*}{ Abbott et al. (1989) } & \multirow{3}{*}{$\begin{array}{l}\mathrm{CCC} / n \text {-hexane-ethyl acetate-methanol- } \\
\text { acetonitrile }(5: 2: 4: 5)\end{array}$} \\
\hline & Lupeol acetate & & \\
\hline & $\beta$-Amyrin acetate & & \\
\hline & Cycloartenyl ferulate & \multirow[t]{2}{*}{ Liu et al. (2013a) } & \multirow[t]{2}{*}{$\mathrm{CCC} / n$-hexane-acetonitrile $(1: 1)$} \\
\hline & $\begin{array}{l}\text { 24-Methylene cycloartanyl } \\
\text { ferulate }\end{array}$ & & \\
\hline & Abrusoside A, B, C, D & Fullas et al. (1990) & $\begin{array}{l}\text { CCC/chloroform-methanol-water } \\
(7: 13: 8)\end{array}$ \\
\hline & No compound names given & Marston et al. (1988) & $\begin{array}{l}\text { CCC/chloroform-methanol-water } \\
(7: 13: 8)\end{array}$ \\
\hline & $\begin{array}{l}\text { Inotodiol } \\
\text { Trametenolic acid }\end{array}$ & Du et al. (2011) & $\begin{array}{l}\mathrm{CCC} / n \text {-hexane-ethyl acetate-methanol- } \\
\text { water }(1: 0.4: 1: 0.4)\end{array}$ \\
\hline & Ursolic acid & \multirow{2}{*}{$\begin{array}{l}\text { Maurya and Srivastava } \\
\text { (2011) }\end{array}$} & \multirow{2}{*}{$\begin{array}{l}\mathrm{CPC} / n \text {-hexane-ethyl acetate-methanol- } \\
\text { water }(1: 2: 1.5: 1) \text { with } 2 \% \text { ammonia } \\
\text { solution in lower aqueous mobile phase } \\
\text { (pH 9.5) }\end{array}$} \\
\hline & Ursolic acid lactone & & \\
\hline & Asiaticoside & \multirow[t]{2}{*}{ Diallo et al. (1991) } & \multirow{2}{*}{$\begin{array}{l}\text { CCC/chloroform- } n \text {-butanol-methanol- } \\
\text { water }(7: 3: 6: 4)\end{array}$} \\
\hline & Madecassoside & & \\
\hline
\end{tabular}


Table 2 continued

\begin{tabular}{|c|c|c|c|}
\hline Classes of compounds & Purified compounds & References & Type of apparatus/solvent system \\
\hline & Taraxeryl acetate & Yang et al. (1995) & CCC/chloroform-methanol-water $(2: 2: 1)$ \\
\hline & Triterpenoic acid & Ito et al. (1990) & CCC/n-hexane-ethanol-water $(6: 5: 2)$ \\
\hline & Acetyl-triterpenoic acid & & \\
\hline & Celastrol & Wu et al. (2004) & $\begin{array}{l}\text { CCC/light petroleum-ethyl acetate- } \\
\text { tetrachloromethane-methanol-water } \\
(1: 1: 8: 6: 1)\end{array}$ \\
\hline & Asiatic acid & Du et al. (2004) & $\mathrm{CCC} / n$-hexane- $n$-butanol- $0.05 \mathrm{M} \mathrm{NaOH}$ \\
\hline & Madecassic acid & & $(5: 1: 6)$ \\
\hline & Asciaticoside & & \\
\hline & Madecassoside & & \\
\hline & Euscaphic acid & Rocha et al. (2007) & CCC/gradient: $n$-hexane-ethyl acetate- \\
\hline & Tormentic acid & & methanol-water $(1: 2: 1.25: 2)$ and $(1: 2: 1.5: 2)$ \\
\hline & $2 \alpha$-Acetyl tormentic acid & & \\
\hline & $3 \beta$-Acetyl tormentic acid & & \\
\hline & Alisol B & Yoon et al. (2009) & CPC/n-hexane-ethyl acetate-methanol- \\
\hline & Alisol B 23-acetate & & water $(10: 2: 10: 7)$ \\
\hline & $\begin{array}{l}\text { Ganoderic acids A, B, C6, D, } \\
\text { E, F, G } \\
\text { Ganoderenic acid D }\end{array}$ & Cheng et al. (2012) & $\begin{array}{l}\text { CCC/stepwise gradient: light petroleum } \\
\text { ether-ethyl acetate-methanol-water } \\
(3: 5: 3: 5) \text { and }(4: 5: 4: 5)\end{array}$ \\
\hline & & & $\begin{array}{l}\text { chloroform-methanol-water } \\
(13: 7: 4)+\text { ammonia }(22 \mathrm{mM}) \text { in aqueous } \\
\text { phase and TFA }(11 \mathrm{mM}) \text { in organic phase } \\
\text { for further purification }\end{array}$ \\
\hline & Dehydrosulphurenic acid & Zhang et al. (2013a) & $\mathrm{CCC} / n$-hexane-ethyl acetate-methanol- \\
\hline & 3-Ketodehydrosulphurenic & & water (1:1.5:1.2:1) \\
\hline & 24-Methylene cycloartanol & Yao et al. (2007) & $\begin{array}{l}\text { CCC/n-hexane-ethyl acetate-acetonitrile } \\
(5: 1: 4)\end{array}$ \\
\hline & Lupenone & Yao et al. (2007) & $\begin{array}{l}\text { CCC/n-hexane-ethyl acetate-acetonitrile } \\
(5: 2: 5)\end{array}$ \\
\hline \multirow{9}{*}{$\begin{array}{l}\text { Triterpenoid saponins } \\
\text { (ginsenosides) }\end{array}$} & $\mathrm{Rc}, \mathrm{Rb} 1$ and $\mathrm{Re}$ & Wang et al. (2010) & CPC/ethyl acetate- $n$-butanol-water $(1: 1: 2)$ \\
\hline & $\mathrm{Rg} 3, \mathrm{Rk} 1, \mathrm{Rg} 5$ and $\mathrm{F} 4$ & Ha et al. (2007) & $\begin{array}{l}\text { CCC/methylene chloride-methanol-water- } \\
\text { isopropanol }(6: 6: 4: 1)\end{array}$ \\
\hline & $\mathrm{Rf}, \mathrm{Rd}, \mathrm{Re}$ and $\mathrm{Rb} 1$ & Qi et al. (2010) & $\begin{array}{l}\mathrm{CCC} / \text { methylene chloride-methanol-5 mM } \\
\text { aqueous ammonium acetate-isopropanol } \\
(6: 2: 4: 3)\end{array}$ \\
\hline & $\operatorname{Re}$ & Engelberth et al. (2010) & CPC/heptane-n-butanol-water $(3: 4: 7)$ \\
\hline & $\begin{array}{l}\mathrm{Rg} 1, \mathrm{Re}, \mathrm{Rf}, \mathrm{Rh} 1, \mathrm{Rb} 1, \mathrm{Rc} \\
\mathrm{Rb} 2 \text { and } \mathrm{Rd}\end{array}$ & Shehzad et al. (2011) & $\begin{array}{l}\text { CCC/chloroform-methanol-water- } \\
\text { isopropanol }(4: 3: 2: 1)\end{array}$ \\
\hline & $\mathrm{Re}, \mathrm{Rb} 1, \mathrm{Rc}$ and $\mathrm{Rb} 2$ & Cheng et al. (2011) & $\begin{array}{l}\text { CCC/methylene chloride-methanol-water- } \\
\text { isopropanol }(6: 2: 4: 3, \mathrm{v} / \mathrm{v}) \text { further } \\
\text { purification of Rb1, Rc and Rb2 in } n- \\
\text { hexane- } n \text {-butanol- } 0.1 \% \text { formic acid } \\
(0.7: 3: 4)\end{array}$ \\
\hline & $\begin{array}{l}\mathrm{Rb} 1, \mathrm{Rb} 2, \mathrm{Rc}, \mathrm{Rd}, \mathrm{Re}, \mathrm{Rg} 1, \\
\mathrm{Rf} \text { and } \mathrm{Rh} 1\end{array}$ & Shehzad et al. (2012) & $\begin{array}{l}\text { CCC/stepwise gradient: } n \text {-hexane-ethyl } \\
\text { acetate-methanol-water }(5: 6: 1: 4) \text { and } \\
(4: 3: 1: 2) \text { and }(3: 3: 1: 2)\end{array}$ \\
\hline & $\operatorname{Re}$ and $\operatorname{Rg} 1$ & Chen et al. (2012) & CCC/ethyl acetate- $n$-butanol-water $(4: 1: 6)$ \\
\hline & $\begin{array}{l}\text { Rh1, Rf, Rd, Rg1, Re, Rc, } \\
\text { Rb2 and Rb1 }\end{array}$ & Shehzad et al. (2013) & $\begin{array}{l}\text { CCC/methylene chloride-methanol-water- } \\
\text { isopropanol }(1: 1: 2: 1)\end{array}$ \\
\hline
\end{tabular}


Table 2 continued

\begin{tabular}{|c|c|c|c|}
\hline $\begin{array}{l}\text { Classes of } \\
\text { compounds }\end{array}$ & Purified compounds & References & Type of apparatus/solvent system \\
\hline & $\begin{array}{l}\text { Rg6, Rg5, Rk1,F4, Rg3, Rg2, Rf, } \\
\text { Rd, Rg1, Re, Rc, Rb2, Rb1 }\end{array}$ & Shehzad et al. (2013) & $\begin{array}{l}\text { CCC/stepwise gradient: methylene chloride- } \\
\text { methanol-water-isopropanol }(5: 4: 1: 3) \text { and } \\
(2: 2: 1: 2)\end{array}$ \\
\hline & $\mathrm{Rb} 1$ and $\mathrm{Rb} 2$ & Wang et al. (2013) & CPC/ethyl acetate- $n$-butanol-water $(0.8: 1.2: 2)$ \\
\hline & $\begin{array}{l}\mathrm{Rg}, \mathrm{Rd}, \mathrm{Re} \text { and } \mathrm{Rb} \\
\text { Notoginsenoside } \mathrm{R}\end{array}$ & Cao et al. (2003) & $\begin{array}{l}\text { CCC/chloroform-2-butanol-methanol-water } \\
(5: 1: 6: 4)\end{array}$ \\
\hline & $\mathrm{Rg}, \mathrm{Rd}, \mathrm{Re}$ and $\mathrm{Rb}$ & Cao et al. (2003) & CCC/ethyl acetate- $n$-butanol-water $(1: 1: 2)$ \\
\hline & Notoginsenoside R & & \\
\hline & $\mathrm{Rg}, \mathrm{Re}$ and $\mathrm{Rb}$ & Du et al. (2003b) & CCC/n-hexane- $n$-butanol-water $(3: 4: 7)$ \\
\hline & Notoginsenoside R & & \\
\hline & Ro & Cheng et al. (2010) & $\begin{array}{l}\text { CCC/ethyl acetate-isopropanol-0.1\% formic acid } \\
(3: 1: 5)\end{array}$ \\
\hline & $\operatorname{Rg} 1, \operatorname{Re}$ and $\mathrm{Rb} 1$ & Wang et al. (2011b) & CPC/ethyl acetate- $n$-butanol-water $(1: 1: 2)$ \\
\hline & Notoginsenoside R1 & & \\
\hline \multirow{27}{*}{$\begin{array}{l}\text { Triterpenoid } \\
\text { saponins }\end{array}$} & Lucyoside Q & Du and Gao (2006) & CCC/chloroform-methanol-water (13:7:8) \\
\hline & Lucyoside $\mathrm{H}$ & & \\
\hline & Astragaloside I & Han et al. (2007) & CCC/stepwise gradient: ethyl acetate-2-propanol- \\
\hline & Astragaloside II & & water $(5: 1: 5)$ and $(50: 1: 50)$ \\
\hline & Lancemaside A & Shirota et al. (2008) & $\mathrm{CPC} / n$-hexane- $n$-butanol-methanol- $0.1 \%$ aqueous \\
\hline & Foetidissimoside A & & formic acid $(3: 4: 1: 6)$ \\
\hline & Astersaponin $\mathrm{Hb}$ & & \\
\hline & Saikosaponins-A & Yoon and Kim (2009) & CPC/ethyl acetate- $n$-butanol-methanol-water \\
\hline & Saikosaponins-C & & $(15: 1: 3: 1)$ \\
\hline & Platycoside E & Han et al. (2009) & CCC/n-hexane- $n$-butanol-water $(1: 40: 20)$ \\
\hline & Deapio-platycoside E & & \\
\hline & Platycodin D3 & Han et al. (2009) & CCC/n-hexane- $n$-butanol-water (1:10:5) \\
\hline & Deapio-platycodin D3 & & \\
\hline & Platycodin D & & \\
\hline & Deapio-platycodin D & & \\
\hline & $2^{\prime \prime}-O$-Acetylplatycodin D & Ha et al. (2011) & CCC/chloroform-methanol-isopropanol-water \\
\hline & $3^{\prime \prime}-O$-Acetylpolygalacin $\mathrm{D}$ & & $(3: 2: 2: 3)$ \\
\hline & $2^{\prime \prime}-O$-Acetylpolygalacin & & \\
\hline & $3^{\prime \prime}-O$-Acetylplatycodin $\mathrm{D}$ & & \\
\hline & Polygalacin D & & \\
\hline & No compound names given & Shi et al. (2007) & $\begin{array}{l}\text { CCC/ethyl acetate- } n \text {-butanol-ethanol- } 0.05 \% \text { TFA } \\
(5: 10: 2: 20)\end{array}$ \\
\hline & Astragaloside IV & Peng et al. (2008b) & CCC/stepwise gradient: $n$-hexane-ethyl acetate- \\
\hline & Astragaloside II & & ethanol-water (1:0.6:0.6:1) and (1:1:1:1) \\
\hline & Astragaloside I & & \\
\hline & Acetylastragaloside I & & \\
\hline & Gypsogenin derivatives & Yao et al. (2008) & CCC $/ n$-hexane- $n$-butanol-methanol- $0.02 \%$ TFA \\
\hline & Dianoside C & & $(1: 9: 1: 9)$ \\
\hline
\end{tabular}


Table 2 continued

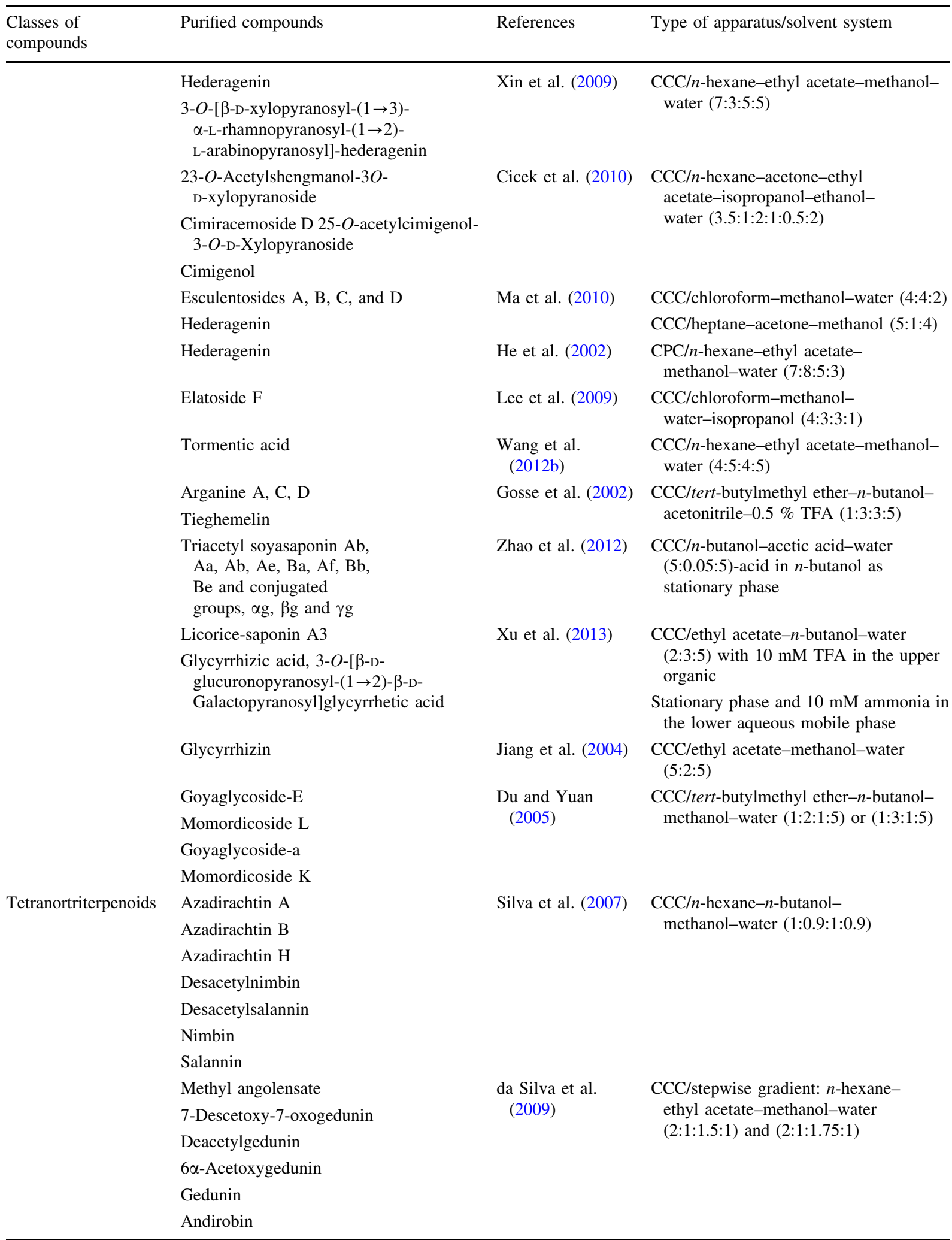


Table 2 continued

\begin{tabular}{|c|c|c|c|}
\hline Classes of compounds & Purified compounds & References & Type of apparatus/solvent system \\
\hline \multirow[t]{19}{*}{$\begin{array}{r}\text { Tetraterpenoids } \\
\text { (Carotenoids) }\end{array}$} & $\begin{array}{l}\text { Cochloxanthin } \\
\text { Dihydrocochloxanthin }\end{array}$ & $\begin{array}{l}\text { Diallo and } \\
\quad \text { Vanhaelen (1988) }\end{array}$ & $\begin{array}{l}\text { CCC/tetrachloromethane-methanol- } \\
\text { water }(5: 4: 1)\end{array}$ \\
\hline & Zeaxanthin & Chen et al. (2005) & $\begin{array}{l}\text { CCC/n-hexane-ethyl acetate-ethanol- } \\
\text { water }(8: 2: 7: 3)\end{array}$ \\
\hline & Crocin & Jiang et al. (2011) & $\begin{array}{l}\mathrm{CCC} \text { /tert-butylmethyl ether- } n \text {-butanol- } \\
\text { acetonitrile-water }(2: 2.5: 1: 5)\end{array}$ \\
\hline & Fucoxanthin & Kim et al. (2011) & $\begin{array}{l}\mathrm{CPC} / n \text {-hexane-ethyl acetate-ethanol- } \\
\text { water }(5: 5: 7: 3)\end{array}$ \\
\hline & Lutein & Wei et al. (2003) & $\begin{array}{l}\text { CCC/n-heptane-chloroform-acetonitrile } \\
(10: 3: 7)\end{array}$ \\
\hline & Zeaxanthin & Aman et al. (2005) & $\mathrm{CCC} / n$-hexane-ethanol-water $(6: 5: 1.3)$ \\
\hline & \multicolumn{3}{|l|}{ Lutein } \\
\hline & Lutein & $\begin{array}{l}\text { Tsao and Yang } \\
\text { (2006) }\end{array}$ & CCC/n-hexane-ethanol-water $(6: 4.5: 1.5)$ \\
\hline & Canthaxanthin & Li et al. (2006) & $\mathrm{CCC} / n$-hexane-ethanol-water $(10: 9: 1)$ \\
\hline & $9^{\prime}$-Cis-neoxanthin & $\begin{array}{l}\text { Baldermann et al. } \\
\text { (2007) }\end{array}$ & $\mathrm{CCC} / n$-hexane-ethanol-water $(5: 5: 4.5)$ \\
\hline & Lycopene & $\begin{array}{l}\text { Baldermann et al. } \\
\text { (2008) }\end{array}$ & $\begin{array}{l}\mathrm{CCC} / n \text {-hexane-dichloromethane- } \\
\text { acetonitrile (30:11:18) with } 85 \mathrm{mg} / \mathrm{L} \text { of } \\
\text { 3-tert-butyl-4-hydroxyanisol and 2-tert- } \\
\text { butyl-p-cresol }\end{array}$ \\
\hline & $\begin{array}{l}\text { Crocins } 1,2,5 \\
\text { Picrocrocin }\end{array}$ & $\begin{array}{l}\text { Lechtenberg et al. } \\
\text { (2008) }\end{array}$ & $\begin{array}{l}\mathrm{CPC} / n \text {-hexane-ethyl acetate-ethanol- } \\
\text { water }(1: 3: 4: 7)\end{array}$ \\
\hline & Fucoxanthin & Xiao et al. (2012) & $\begin{array}{l}\mathrm{CCC} / n \text {-hexane-ethyl acetate-ethanol- } \\
\text { water }(5: 5: 6: 4)\end{array}$ \\
\hline & Lutein & Li et al. (2001) & $\mathrm{CCC} / n$-hexane-ethanol-water $(4: 3: 1)$ \\
\hline & Lycopene & Wei et al. (2001) & $\begin{array}{l}\mathrm{CCC} / n \text {-hexane-dichloromethane- } \\
\text { acetonitrile }(10: 3.5: 6.5)\end{array}$ \\
\hline & Astaxanthin & Li and Chen (2001a) & $\begin{array}{l}\mathrm{CCC} / n \text {-hexane-ethyl acetate-ethanol- } \\
\text { water }(5: 5: 6.5: 3)\end{array}$ \\
\hline & Tephrosin & \multirow[t]{3}{*}{ Ye et al. (2008a) } & \multirow{3}{*}{$\begin{array}{l}\mathrm{CCC} / n \text {-hexane-ethyl acetate-methanol- } \\
\text { water }(1: 0.8: 1: 0.6)\end{array}$} \\
\hline & Deguelin & & \\
\hline & 6a,12a-Dehydrodeguelin & & \\
\hline
\end{tabular}

$C C C$ counter-current chromatography, $C P C$ centrifugal partition chromatography

trachelosperogenin E and sericoside was achieved using a three-phase solvent system composed of $n$ heptane, tert-butylmethyl ether, acetonitrile and water. After mixing all solvents in equal volume, the upper phase was separated and tert-butylmethyl ether was shaken with the remaining two-phase solvent system in order to decrease the polarity. The lower phase was used as a stationary phase. Sericic acid was identified in the fractions collected when the upper phase was used as the mobile phase, while the elution of the middle phase led to the separation of trachelosperogenin $E$ and sericoside (Hamzaoui et al. 2013).
Zhao and Du (2007) proposed a novel non-aqueous two-phase solvent system composed of sunflower oil and ethanol to separate solanesol-a non-cyclic terpene alcohol used as a food additive for preventing cardiac arrest and cancer. Before, this target molecule was purified from tobacco leaves extract with petroleum and methanol, both solvents being unsuitable for the food industry.

Sometimes the addition of a small amount of acid can significantly improve the purification. Shirota et al. (2008) purified the saponins lancemaside A, foetidissimoside $\mathrm{A}$ and astersaponin $\mathrm{Hb}$ from a hot 
water extract of Codonopsis lanceolata roots used CPC with mixture of $n$-hexane- $n$-butanol-methanol$0.1 \%$ aqueous formic acid (3:4:1:6) as the two-phase solvent system. Because the crude extract contained large amounts of sugars, an aqueous phase was chosen as the stationary phase so that these sugars would be retained in the partition cells, whereas an organic phase was chosen as mobile phase for elution of the target compound. Addition of formic acid to the twophase solvent system retained the carboxy group in the non-ionised form and allowed the separation of the lancemasides from the large amounts of water-soluble sugars present. The addition of acetic acid to the ethyl acetate- $n$-butanol-water mixture improved the resolution of soyasaponins, naturally occurring triterpenoid glycosides. A suitable mixture $n$-butanol, acetic acid, water (5:0.05:5) was taken but acetic acid was added only in $n$-butanol as the stationary phase (Zhao et al. 2012). During the separation, with the movement of the mobile phase the acetic acid was gradually diluted with water and the polarity of the stationary phase changed as a gradient. It is worth mentioning that the main difficulties with soyasaponin isolation and purification are that the soyasaponins coexist in soybeans with the isoflavone glycosides and they share overlapping polarities. There is also the structural similarity of soyasaponin compounds.

$\mathrm{CCC}$ is popularly applied for purification of ginsenosides, an important group of terpenoids due to their biological activity. They generally fall in the moderate to polar category and therefore require similar solvent systems for purification (Qi et al. 2010; Shehzad et al. 2013). A good solubility for saponin-like compounds can be provided by the addition of chloroform or other chlorinated solvents and a gradual polarity change between phases can be achieved by varying the methanol ratio in the system or by adding another alcohol like isopropanol (Qi et al. 2010). Shehzad et al. (2013) proposed an efficient CCC separation method in which a flow-rate gradient technique was coupled with a new solvent gradient dilution strategy for the isolation of ginsenosides from Korean red ginseng (steam-treated $P$. ginseng). The column was first entirely filled with the upper stationary phase mixture of methylene chloride-methanol-isopropanol-water $(5: 4: 1: 3)$ in a reversed phase system. After $300 \mathrm{~min}$, when five ginsenosides had eluted, the flow rate was increased from 1 to $1.2 \mathrm{ml} / \mathrm{min}$ and also the dilution of the lower phase was initiated and was changed to
$100 \%$ of lower phase composed of the mixture mentioned above in a ratio (2:2:1:2). Overall, 13 ginsenosides including $\mathrm{Rg} 1, \mathrm{Re}, \mathrm{Rf}, \mathrm{Rg} 2, \mathrm{Rb} 1, \mathrm{Rb} 2$, Rc, Rd, Rg3, Rk1, Rg5, Rg6, and F4 were purified (Shehzad et al. 2013). Qi et al. (2010) for the purification of ginsenosides $\mathrm{Rf}, \mathrm{Rd}, \mathrm{Re}$ and $\mathrm{Rb} 1$, applied mixtures of methylene chloride, methanol, water and isopropanol (6:2:4:3).

Emulsification can often be a problem in the CCC purification of ginsenosides. Generally, adding an electrolyte, such as salt or acid, can help to eliminate such emulsification. Salt is not recommended because an additional desalting process will be necessary after the separation. Therefore, formic acid is often chosen to prevent emulsification (Cheng et al. 2010). However, Qi et al. (2010) preferred to avoid an acidic environment, which would lead to the decomposition of ginsenosides. Instead, the inorganic salt ammonium acetate was chosen, because it is volatile and can be precipitated in warm acetone for sample recovery. The addition of this salt resulted in a very slight decrease in $K$ values and thus a shorter separation time. In the end, the proposed solvent system was methylene chloridemethanol-5 $\mathrm{mg} / \mathrm{mL}$ aqueous ammonium acetate-isopropanol (6:2:4:3).

As the solvent system contained methylene chloride and methanol, both toxic to humans and the environment, Wang et al. (2010) chose low toxic solvents such as ethyl acetate and $n$-butanol. Since the ginsenosides in $P$. quinquefolium L. have a comparatively larger polarity with solubility in hydrophilic solvents, both phases require a certain hydrophilicity to get a good separation. Initially, the hexane- $n$ butanol-water solvent systems were scanned at several volume ratios, but all the systems had a poor retention in the CPC column. Then ethyl acetate was then employed instead of hexane in order to enhance the retention of the stationary phase in the column. Because ginsenosides are easily dissolved in $n$-butanol, and the viscosity of $n$-butanol is comparatively large, so in an ethyl acetate- $n$-butanol-water solvent system, the addition of $n$-butanol could delay the peak elution time and affect the separation. Overall, the system of ethyl acetate- $n$-butanol-water (1:1:2) was used for successful separation of three ginsenosides $\mathrm{Rc}, \mathrm{Rb} 1$, and Re.

Counter-current chromatography has also been used for the separation of minor and structurally similar compounds. Fan and He (2006) using 
HSCCC with $n$-hexane-ethyl acetate-methanol-water (3:6:4:2) to separate not only ursolic acid, which is a very small content of the leaves of Diospyros kaki, but also two other pentacyclic triterpenes: barbinervic acid and its epimer rotungenic acid, differing only with the configuration of a hydroxyl group at position C3: one contains an axial and the other an equatorial hydroxyl group. HSCCC was also employed for the separation and purification of minor constituents in Platycodi Radix. Platycosides, the saponins that are the major active constituents, are typically composed of oleanene backbones with two side chains; one a glucose unit attached through an ether linkage at the $\mathrm{C}-3$ position of a triterpene, and the other 28- $O$ arabinoserhamnose-xylose-apiose linked by an ester bond. They also have different substituents at the C-4 position. Because the content of these compounds is very low, conventional methods are frequently not suitable for the separation. Ha et al. (2011) used a twophase solvent system consisting of chloroform-methanol-isopropanol-water $(3: 2: 2: 3)$ for purification of minor saponins $2^{\prime \prime}$ - $O$-acetylplatycodin D, $3^{\prime \prime}$ - $O$-acetylpolygalacin $\mathrm{D}, 2^{\prime \prime}$ - $O$-acetylpolygalacin and a mixture of $3^{\prime \prime}-O$-acetylplatycodin $\mathrm{D}$ and polygalacin $\mathrm{D}$, which were further successful purified using prepHPLC (Ha et al. 2011). Ha and Kim (2009) also used HSCCC for the separation of three pairs of platycosides and their deapiose forms. They used an interesting modification: the column was first filled with a mixture of the two phases, thus reducing the amount of time for hydrodynamic equilibrium to be established. The ratio of two phases was optimised at 70:30 (stationary phase-mobile phase) based on the amount of time required to reach hydrodynamic equilibrium. A series of solvent systems were tested. In the gradient elution mode, the retention of the stationary phase was extremely low. The authors decided to perform the separation in two stages. First, platycoside E, deapioplatycoside $\mathrm{E}$, a mixed fraction containing platyodin $\mathrm{D}$ and deapiose form, and a fraction containing platycodin D3 and deapiose form were separated using the $n$ hexane-butanol-water (1:40:20) in reversed-mode. Then mixed fractions I and II were further purified in the normal elution mode with the above mentioned solvent system in the ratio 1:10:5.

CCC/CPC seems to be one of the few efficient approaches for the separation of xanthanolides, a bicyclic subtype of sesquiterpenic lactones characterized by a 5,7-fused system containing a $\gamma$-lactone moiety. Because of some well documented and promising activity purification of these compounds is important. The presence of several chiral centres probably explain the difficulty obtaining enantiomericaly pure xanthanolides by total synthesis, thus purification from natural source seems to be the best solution. Xanthonolides are coextracted with chlorophyll and lipids. The pigment crystallization and the delipidation in order to clean the extract is not selective and cause the partial loss of target compounds. Purification of several xanthanolides (xanthathin, 4-epi-xanthanol and 4-epi-isoxanthanol) was realized in one step, directly from the crude chloroformic extract of the leaves of $X$. macrocarpum with a mixture of heptane-ethyl acetate-methanol-water (1:1:1:1) (Pinel et al. 2007).

High-speed counter-current chromatography can be applied as a method suitable for fingerprinting. $\mathrm{Gu}$ et al. (2006) used it for quality control of TCMs and identification of the active compounds of Salvia miltiorrhiza Bunge, a popular traditional Chinese medicine. In order to purify a series of tanshinones a stepwise elution with solvent systems composed of $n$ hexane-ethanol-water (10:5.5:4.5) and (10:7:3) was used. The method was compared with more conventional approaches, such as high performance liquid chromatography (HPLC), high performance capillary electrophoresis (HPCE), and thin-layer chromatography scan (TLCS). In the HSCCC separation, 12 components were separated, with good resolution and precision, within $13 \mathrm{~h}$. HSCCC showed better performance in the analysis of tanshinones, which produced a fingerprint which contained more chemical information than that of e.g. TLCS.

$\mathrm{Lu}$ et al. (2007) proposed this effective twodimensional counter-current chromatographic method for the simultaneous isolation and purification of oridonin and ponicidin from a crude extract of Rabdosia rubescens with a pair of two-phase solvent systems composed of $n$-hexane-ethyl acetate-methanol-water (1:5:1:5 and 3:5:3:5, v/v). A combination of stepwise CCC and pH-zone-refining is also possible. Cheng et al. (2012) reported its successful combination in the separation of the main components from $G$. lucidum. In the first step, a two-phase solvent system composed of petroleum ether-ethyl acetate-methanol-water (3:5:3:5 and 4:5:4:5) led to the separation of ganoderic acids GE, GC6 and GF with high purity in one run. Also two peaks containing GG, GB, GA and 
GED, GD, respectively, were collected and their separation was followed by $\mathrm{pH}-$ zone-refining $\mathrm{CCC}$. Chloroform-methanol-water (13:7:4) with $\mathrm{NH}_{4} \mathrm{OH}$ in upper aqueous stationary phase and trifluoroacetic acid as the eluter acid was the most suitable. In another example of two-dimensional CCC, a three-step gradient elution and two-step flow-rate gradient elution was applied to separate 8 diterpene compounds within $80 \mathrm{~min}$ in a single run from the alcohol extract of Pseudolarix kaempferi. Pseudolaric acid B $O$ - $\beta$-Dglucopyranoside, pseudolaric acid $\mathrm{C}$, deacetylpseudolaric acid A, pseudolaric acid A $O$ - $\beta$-D-glucopyranoside, pseudolaric acid B, pseudolaric acid B methyl ester, pseudolaric acid $\mathrm{A}$ and pseudolaric acid $\mathrm{H}$ were obtained with very high purity (He et al. 2012). The separation was performed in normal phase system. In the first step, the mobile phase, composed of hexaneethyl acetate-methanol-water (1:1:1:1), was used with a flow rate $0.5 \mathrm{ml} / \mathrm{min}$. While the mobile phase (upper) of (2:3:2:3) and (1:3.5:1:3.5) were used in the second and third step, and the flow rate of mobile phase was set at $1 \mathrm{ml} / \mathrm{min}$. When the separation was performed in preparative conditions, the initial flow rate of mobile phase was $25 \mathrm{ml} / \mathrm{min}$ in the first step and then increased to $50 \mathrm{ml} / \mathrm{min}$ in the second and third step.

\section{Principle advantages and drawbacks of CCC in the purification of natural products}

Counter-current chromatography has a number of key advantages in the purification of natural products. For example, the solvent usage is generally far lower than that of solid phase chromatography systems operating at the same scale (about $25 \%$ ) (Graham et al. 2001). Furthermore, since the process is frequently an isocratic one, a simple analysis of solvent composition allows the recycling of the solvents, reducing the usage still further (Garrard et al. 2007). The technique also allows for $100 \%$ recovery of the sample components. In other words, the target compound can always be retrieved since there is no solid phase and therefore no possibility of losses arising from irreversible adsorption onto the solid matrix. This is a significant advantage in every purification process. As a solidfree and therefore relatively gentle chromatographic technique, CCC can be used for the isolation of unstable natural compounds. Baldermann et al. (2007) presented the successful purification of 9'-cis-neoxanthin, the predominant isomer of neoxanthin in green vegetables. Major problems during its isolation include isomerization and oxidation, mainly caused by higher temperatures, light or oxygen exposure. When typical solid stationary phase techniques are applied, the rearrangement products can be detected or complete isomerization can be observed. A solid-free technique is a very practical solution for avoiding the above mentioned complications.

Particulates, such as cell debris, are generally well tolerated in CCC, particularly when performed at large scale where the tubing bore may be up to $10 \mathrm{~mm}$ in diameter. This is another major advantage over solid phase chromatography systems. Thus filtering a sample is frequently not necessary, depending on the scale of CCC employed, and even if it is required, it is usually a simple filter paper filtration. With processing times similar to that of other purification methods, scale up is also possible with modern instruments with examples existing running from milligram to kilogram levels (Garrard et al. 2008). The technique can be operated in normal batch injection mode, or as a continuous extraction process for better throughput. A wide range of polarities can be processed due to the range of solvents that may be used (the literature reports examples with a $\log \mathrm{P}$ range from -4.7 (colistin peptide antibiotic) (Ikai et al. 1998) to +17.6 (lycopene) (Wei et al. 2001). Also, the separation of compounds with vastly different polarities from a single extract is possible. For example, in order to purify a wide range of polarity of triterpene saponis from Panax notoginseng, Zhang et al. (2013b) successfully coupled accelerated solvent extraction (ASE) and high-performance counter-current chromatography (HPCCC). First the upper phase of the solvent system ethyl acetate- $n$-butanol-water (1:1:2 or $1.2: 1: 2)$ or ethyl acetate- $n$-butanol-methanolwater $(3: 5: 1.5: 6)$ was used as both the ASE solvent and HPCCC stationary phase. The polar saponis were eluted. In order to separate fractions with moderate polarity, the upper phase of system ethyl acetate- $n$ butanol-methanol-water $(6: 3: 2: 6$ or $7: 3: 2: 7)$ was used. Finally, the upper phase of the solvent system of $n$-hexane- $n$-butanol-methanol-water $(8: 2: 2: 8)$ or $n$-hexane-ethyl acetate- $n$-butanol-methanol-water (0.2:10:0.5:1.5:8) was used as both the ASE solvent and HPCCC stationary phase to elute the low polar compounds. This combination of methods allowed the 
purification of notoginsenosides R6, R1, Spt1 and ginsenosides Rb1, F4, Rh3, Rg3, Rs3 and Rk1 with a wide range of polarity (Zhang et al. 2013b).

Compared to the early instruments, the quality of modern CCC apparatus is very good. In most cases the coils are tough and the machinery robust. A set of coils would be expected to last the lifetime of the centrifuge and maintenance and running costs are low. Unlike solid phase chromatography, there is no change to component retention over time (no column aging effects) as a freshly-filled coil of solvents is used each run. This makes it easier to consistently satisfy current regulatory requirements when performing purifications under a good laboratory practice (GLP) or good manufacturing practice (GMP) environment.

As has been seen in a number of quoted examples, a large advantage of CCC is that the technique can be operated in a number of different modes, since both the mobile and the stationary phase are liquid. Either normal or reverse phase chromatography can be chosen, depending on which solvent phase is selected to be the mobile one. However, it is even possible to switch from normal phase elution to reverse phase (or vice versa) in the middle of a run. Intermittent countercurrent extraction (ICCE) is a continuous process where the operation alternates between normal and reverse phase mode at regular intervals, with the sample continuously introduced in the middle of the coil (Hewitson et al. 2009). In the reference quoted, this technique was successfully used to purify a diterpenoid, triptolide, a high value target compound, from a Chinese herbal plant, from $2 \%$ in the crude extract to over $98 \%$ purity. This was achieved by retaining and enriching the target compound within the CCC coil while washing away all the other components of the crude material. Alternatively, components can be recovered by eluting the liquid stationary phase without any compound losses whilst maintaining resolution (Berthod et al. 2003), a technique known as elution-extrusion. This technique is frequently adopted at the end of standard CCC purification runs simply to ensure that no loss of target compound has occurred within the liquid stationary phase. Another possibility is co-current chromatography (Berthod and Hassoun 2006) where both phases are pumped in the same direction. All of these options use a conventional CCC centrifuge and are thus easy to implement in the laboratory or pilot plant. However, with modifications to the CCC coils, continuous counter-current extraction (CCCE) is possible, where one phase is pumped in the opposite direction to the other and the sample is introduced continuously into the centre of the coil (Ito et al. 2006; Van den Heuvel 2007).

The method also allows a two dimensional procedure to be applied. The purification of very similar terpene lactones from G. biloba $\mathrm{L}$, such as bilobalide, ginkgolides $\mathrm{A}, \mathrm{B}, \mathrm{C}$, and $\mathrm{J}$, is an example. The partitioning experiment assisted the design of a $2 \mathrm{D}$ procedure using a pair of orthogonal solvent systems: chloroform-methanol-water (10:7:3) and hexaneethyl acetate-methanol-water (4:6:4:6) with addition of $0.5 \%$ DMSO to increase the resolution of ginkgolides A and B. This approach separated these almost equipolar lactones (Qiu et al. 2012).

The main drawbacks of the technique, particularly when compared to preparative HPLC and other solid phase chromatography techniques, include a lower efficiency. When measured in terms of theoretical plates, the efficiency of a "good" CCC apparatus is in the low thousand plate range. This figure cannot be directly compared to HPLC plate counts due to the much higher percentage of stationary phase (around $80 \%$ compared to perhaps $5 \%$ active stationary phase sites in HPLC) and the resolution of CCC can be extremely good. However, a low efficiency results in broad peaks, making the technique far more suited to a preparative application as opposed to an analytical one. Also the narrow polarity range within each run should be emphasised. As mentioned above, a wide range of polarities can be processed by CCC by using different solvent systems. However, a single CCC run operates over a relatively narrow polarity window. Although some examples of gradient elution have been reported (see Table 2), these do not give as wide a polarity range as that achievable with HPLC. The narrow polarity window can be used to advantage when it is desired to pluck a single target compound from a complex mixture. However, it is a disadvantage when a dozen pure compounds are required from the mixture in a single purification run. The CCC apparatus does not inherently lend itself to easy automation and thus its operation can appear labour-intensive. In addition, the instruments have not received the same intensive commercial development that has made modern HPLC and GC equipment so sophisticated. This situation will undoubtedly improve in the future, with advances in CCC machine design. Finally, the 
solvent system selection is undoubtedly time-consuming. As the two phases are liquid, changes in one phase directly affects the other. Analysis of a range of possible solvent systems can be performed by an automated liquid-handling robot but this still requires a number of hours to complete. This is a current area of research in the field and no doubt large improvements in solvent system selection time can be expected over the next few years.

\section{Conclusions}

Although initially dismissed by many chemists and purification scientists as slow, unreliable and temperamental, steady development of the technique of CCC on both the engineering and the application side has transformed it into a technique worthy of inclusion in the natural product scientist's arsenal. On one side, engineering developments have produced machines that are robust, capable of fast, efficient separations and able to accept high injection loadings. On the other side, developments in the application protocols have produced modes of operation and solvent systems to purify out compounds from the full polarity spectrum. Combined, these have produced a technique that is wonderfully suited to natural product purifications, particularly on a large preparative scale, with advantages over solid phase techniques such as the ability to accept particulates and to always recover all components, and advantages over the old-style liquid-liquid techniques such as high speed, high loading and high resolution. To assist users of CCC and those wishing to experiment with the technique, a comprehensive phase selection table has been generated by distilling the solvent system information presented from CCC terpenoids purifications over the last 30 years.

Open Access This article is distributed under the terms of the Creative Commons Attribution License which permits any use, distribution, and reproduction in any medium, provided the original author(s) and the source are credited.

\section{References}

Abbott T, Peterson R, McAlpine J et al (1989) Comparing centrifugal countercurrent chromatography, nonaqueous reversed phase HPLC and AG ion exchange HPLC for the separation and characterization of triterpene acetates. J Liq Chromatogr Relat Technol 12:2281-2301
Acton N, Klayman DL, Rollman IJ et al (1986) Isolation of artemisinin (ginghaosu) and its separation from artimisitene using the Ito multilayer coil separator-extractor and isolation of arteannuin B. J Chromatogr 355:448-450

Agostinho D, Boudesocque L, Thery-Kone I et al (2013) A new meroterpenoid isolated from roots of Ptaeroxylon obliquum Radlk. Phytochem Lett 6:560-566

Ajikumar PK, Tyo K, Carlsen S et al (2008) Terpenoids: opportunities for biosynthesis of natural product drugs using engineered microorganisms. Mol Pharm 5:167-190

Aman R, Carle R, Conrad J et al (2005) Isolation of carotenoids from plant materials and dietary supplements by high-speed counter-current chromatography. J Chromatogr A 1074: 99-105

Baldermann S, Reinhard A, Köhler N et al (2007) Application of high-speed counter-current chromatography for the isolation of $9^{\prime}$-cis-neoxanthin from fresh spinach. J Chromatogr A 1151:183-186

Baldermann S, Ropeter K, Köhler N et al (2008) Isolation of alltrans lycopene by high-speed counter-current chromatography using a temperature-controlled solvent system. J Chromatogr A 192:191-193

Berthod A, Hassoun M (2006) Using the liquid nature of the stationary phase in countercurrent chromatography: IV. The cocurrent CCC method. J Chromatogr A 1116:143-148

Berthod A, Ruiz-Angel MJ, Carda-Broch S (2003) Elutionextrusion countercurrent chromatography: use of the liquid nature of the stationary phase to extend the hydrophobicity window. Anal Chem 75:5508-5517

Brown GD (2010) The biosynthesis of artemisinin (Qinghaosu) and the phytochemistry of Artemisia annua L. (Qinghao). Molecules 15:7603-7698

Cao X, Tian Y, Zhang TY, Liu QH et al (2003) Separation of dammarane-saponins from notoginseng, root of Panax notoginseng (Burk.) F.H.Chen by HSCCC coupled with evaporative light scattering detector. J Liq Chromatogr Relat Technol 26:1579-1591

Chen F, Lu HT, Jiang Y (2004) Purification of paeoniflorin from Paeonia lactiflora Pall. by high speed counter current chromatography. J Chromatogr A 1040:205-208

Chen F, Li HB, Wong R et al (2005) Isolation and purification of the bioactive carotenoid zeaxanthin from the microalga Microcystis aeruginosa by high-speed counter-current chromatography. J Chromatogr A 1064:183-186

Chen Q, Hu X, Li J et al (2011) Preparative isolation and purification of cuminaldehyde and p-menta-1,4-dien-7-al from the essential oil of Cuminum cyminum L. by highspeed counter-current chromatography. Anal Chim Acta 689:149-154

Chen F, Luo J, Kong L (2012) Fast isolation of ginsenosides Re and $\mathrm{Rg} 1$ from the roots of Panax ginseng by HSCCCELSD combined with MCI gel CC guided by HPLC-MS. J Liq Chromatogr Relat Technol 35:912-923

Cheng Y, Liang Q, Hu P et al (2010) Combination of normal-phase medium-pressure liquid chromatography and high-performance counter-current chromatography for preparation of ginsenoside-Ro from Panax ginseng with high recovery and efficiency. Sep Purif Technol 73:397-402

Cheng Y, Zhang M, Liang Q et al (2011) Two-step preparation of ginsenoside-Re, Rb1, Rc and Rb2 from the root of Panax 
ginseng by high-performance counter-current chromatography. Sep Purif Technol 77:347-354

Cheng CR, Li YF, Xu PP (2012) Preparative isolation of triterpenoids from Ganoderma lucidum by counter-current chromatography combined with $\mathrm{pH}$-zone-refining. Food Chem 130:1010-1016

Cicek SS, Schwaiger S, Ellmerer EP et al (2010) Development of a fast and convenient method for the isolation of triterpene saponins from Actaea racemosa by high-speed countercurrent chromatography coupled with evaporative light scattering detection. Planta Med 76:467-473

da Silva VP, Oliveira RR, Figueiredo MR (2009) Isolation of limonoids from seeds of Carapa guianensis Aublet (Meliaceae) by high-speed countercurrent chromatography. Phytochem Anal 20:77-81

Dang YY, Li XC, Zhang QW et al (2010) Preparative isolation and purification of six volatile compounds from essential oil of Curcuma wenyujin using high-performance centrifugal partition chromatography. J Sep Sci 33:1658-1664

De Souza PA, Rangel LP, Oigman SS et al (2010) Isolation of two bioactive diterpenic acids from Copaifera glycycarpa oleoresin by high-speed counter-current chromatography. Phytochem Anal 21:539-543

Diallo B, Vanhaelen M (1988) Large scale purification of apocarotenoids from Cocleospermum tinctorium by counter-current chromatography. J Liq Chromatogr 11:227-231

Diallo B, Vanhaelen Fastre R, Vanhaelen M (1991) Direct coupling of high speed counter current chromatography to thin layer chromatography. Application to the separation of asiaticoside and madecassoside from Centella asiatica. J Chromatogr 558:446-450

dos Santos BCB, da Silva JCT, Guerrero PG Jr et al (2009) Isolation of chavibetol from essential oil of Pimenta pseudocaryophyllus leaf by high-speed counter-current chromatography. J Chromatogr A 1216:4303-4306

Du Q, Gao S (2006) Preparative separation of saponins from the Luffa cylindeica (L.) Roem. by slow rotary countercurrent chromatography. J Liq Chromatogr Relat Technol 29: 2451-2456

Du Q, Yuan J (2005) Preparation of triterpene saponins from the fruit of L. by high speed countercurrent chromatography (HSCCC). J Liq Chromatogr Relat Technol 28:1717-1724

Du Q, Xiong X, Ito Y (1995) Separation of bioactive quadriterpenic acids from the fruit of Ligustrum lucidum Ait by high-speed countercurrent chromatography. J Liq Chromatogr 18:1997-2004

Du Q, Jerz G, Waibel R et al (2003a) Isolation of dammarane saponins from Panax notoginseng by high speed counter current chromatography. J Chromatogr A 1008:173-180

Du Q, Jerz G, Winterhalter P (2003b) Separation of andrographolide and neoandrographolide from the leaves of Andrographis paniculata using high speed counter current chromatography. J Chromatogr A 984:147-151

Du Q, Jerz G, Chen P et al (2004) Preparation of ursane triterpenoids from Centella asiatica using high speed countercurrent chromatography with step-gradient elution. J Liq Chromatogr Relat Technol 27:2201-2215

Du Q, Daijie W, Ito Y (2006) Preparation of solanesol from a tobacco leaf extract using high speed countercurrent chromatography. J Liq Chromatogr Relat Technol 29:2587-2592
Du D, Zhu F, Chen X et al (2011) Rapid isolation and purification of inotodiol and trametenolic acid from Inonotus obliquus by high-speed counter-current chromatography with evaporative light scatting detection. Phytochem Anal 22:419-423

Engelberth AS, Clausen EC, Carrier DJ (2010) Comparing extraction methods to recover ginseng saponins from American ginseng (Panax quinquefolium), followed by purification using fast centrifugal partition chromatography with HPLC verification. Sep Purif Technol 72:1-6

Fan JP, He CH (2006) Single-step preparative separation of barbinervic acid and its epimer (rotungenic acid), along with two other pentacyclic triterpene acids from the leaves of Diospyros kaki using HSCCC. J Liq Chromatogr Relat Technol 29:815-826

Fischedick JT, Standiford M, Johnson DA et al (2012) Activation of antioxidant response element in mouse primary cortical cultures with sesquiterpene lactones isolated from Tanacetum parthenium. Planta Med 78: 1725-1730

Fischedick JT, Pesic M, Podolski-Renic A et al (2013a) Cytotoxic activity of sesquiterpene lactones from Inula britannica on human cancer cell lines. Phytochem Lett 6: 246-252

Fischedick JT, Standiford M, Johnson DA et al (2013b) Structure activity relationship of phenolic diterpenes from $\mathrm{Sal}$ via officinalis as activators of the nuclear factor E2-related factor 2 pathway. Bioorgan Med Chem 21:2618-2622

Fischer N, Weinreich B, Nitz S et al (1991) Applications of high speed counter current chromatography for the separation and isolation of natural products. J Chromatogr 538: 193-202

Fisher D, Garrard IJ, van den Heuvel R et al (2005) Technology transfer and scale up of a potential cancer preventative plant dynamic extraction of glucoraphanin. J Liq Chromatogr Relat Technol 28:1913-1922

Frighetto N, Welendorf RM, Pereira da Silva AM et al (2005) Purification of betulinic acid from Eugenia florida (Myrtaceae) by high-speed counter-current chromatography. Phytochem Anal 16:411-414

Frighetto RTS, Welendorf RM, Nigro EN et al (2008) Isolation of ursolic acid from apple peels by high speed countercurrent chromatography. Food Chem 106:767-771

Fullas F, Choi YH, Kinghorn D et al (1990) Sweet-tasting triterpene glycoside constituents of Abrus fruticulosus. Planta Med 56:332-333

Garrard IJ (2005) Simple approach to the development of a CCC solvent selection protocol suitable for automation. J Liq Chromatogr Relat Technol 28:1923-1935

Garrard IJ, Janaway L, Fisher D (2007) Minimising solvent usage in high speed, high loading and high resolution isocratic dynamic extraction. J Liq Chromatogr Relat Technol 30:151-163

Garrard IJ, Fisher D, Sutherland IA (2008) Dynamic extraction: a high speed, high capacity purification process that is rapidly scaleable. LC/GC N Am 26:2-7

Gasper MP, Berthod A, Talabardon K et al (1995) An evaluation of the differential partitioning and separation of C60 and C70 fullerenes in a biphasic system using centrifugal partition chromatography. J Liq Chromatogr Relat Technol 18:1019-1034 
Geng Y, Liu J, Lv R et al (2007) An efficient method for extraction, separation and purification of eugenol from Eugenia caryophyllata by supercritical fluid extraction and high-speed counter-current chromatography. Sep Purif Technol 57:237-241

Gosse BK, Gnabre JN, Ito Y et al (2002) Isolation of saponins with viral entry inhibitory activity by combined chromatographic methods. J Liq Chromatogr Relat Technol 25:3199-3211

Graham AS, McConvey IF, Shering P (2001) An evaluation of the performance of a preparative CCC machind for the separation of an active pharmaceutical ingredient. J Liq Chromatogr Relat Technol 24:1811-1825

Grancher D, Jaussaud P, Durix A et al (2004) Countercurrent chromatographic isolation of lolitrem $\mathrm{B}$ from endophyte infected ryegrass (Lolium perenne $\mathrm{L}$ ) seed. J Chromatogr A 159:73-81

Graziose R, Rathinasabapathy T, Lategan C et al (2011) Antiplasmodial activity of aporphine alkaloids and sesquiterpene lactones from Liriodendron tulipifera $L$. J Ethnopharmacol 133:26-30

Gu M, Zhang G, Su Z et al (2004) Identification of major active constituents in the fingerprint of Salvia miltiorrhiza Bunge developed by high speed counter current chromatography. J Chromatogr A 1041:239-243

Gu M, Su Z, Ouyang F (2006) Fingerprinting of Salvia miltiorrhiza Bunge by thin-layer chromatography scan compared with high speed countercurrent chromatography. J Liq Chromatogr Relat Technol 29:1503-1514

Gutiérrez F, Estévez-Braun A, Ravelo AG et al (2007) Terpenoids from the medicinal plant Maytenus ilicifolia. J Nat Prod 70:1049-1052

Ha YW, Kim YS (2009) Preparative isolation of six major saponins from Platycodi radix by high-speed counter-current chromatography. Phytochem Anal 20:207-213

Ha YW, Lim SS, Ha IJ et al (2007) Preparative isolation of four ginsenosides from Korean red ginseng (steam-treated Panax ginseng C. A. Meyer), by high-speed counter-current chromatography coupled with evaporative light scattering detection. J Chromatogr A 1151:37-44

Ha IJ, Kang M, Na YC et al (2011) Preparative separation of minor saponins from Platycodi Radix by high-speed counter-current chromatography. J Sep Sci 34:2559-2565

Hamzaoui M, Renault JH, Nuzillard JM et al (2013) Stepwise elution of a three-phase solvent system in centrifugal partition extraction: a new strategy for the fractionation and phytochemical screening of a crude bark e xtract. Phytochem Anal 24:367-373

Han X, Zhang T, Ito Y (2003) Separation of high purity przewaquinone A by high speed countercurrent chromatography. J Liq Chromatogr Relat Technol 26:1267-1274

Han QB, Song JZ, Qiao CF et al (2007) Preparative isolation of cyclolanostane-type saponins from Astragalus membranaceus Bge. var. mongholicus (Bge.) Hsiao by TLC-MS/ MS guided high-speed counter-current chromatography. J Sep Sci 30:135-140

Han QB, Wong L, Yang NY et al (2008) A simple method to optimize the HSCCC two-phase solvent system by predicting the partition coefficient for target compound. J Sep Sci 31:1189-1194
Han QB, Wong L, Lai F et al (2009) Preparative isolation of pseudolaric acids A and B and their glucosides from the root bark of Pseudolarix kaempferi using high-speed counter-current chromatography. J Sep Sci 32:309-313

He W, Van Puyvelde L, Maes L et al (2002) Antitrichomonas in vitro activity of Cussonia holstii Engl. Nat Prod Res 17:127-133

He F, Bai Y, Wang J et al (2011) Isolation and purification of oridonin from the whole plant of Isodon rubescens by highspeed counter-current chromatography. Molecules 16: 7949-7957

He S, Li S, Yang J et al (2012) Application of step-wise gradient high-performance counter-current chromatography for rapid preparative separation and purification of diterpene components from Pseudolarix kaempferi Gordon. J Chromatogr A 1235:34-38

Hewitson P, Ignatova S, Ye H et al (2009) Intermittent countercurrent extraction as an alternative approach to purification of Chinese herbal medicines. J Chromatogr A 1216: $4187-4192$

Hu J, Liang Y, Xie Y et al (2007) Isolation and purification of solanesol from potato leaves by high-speed counter-current chromatography and identification by atmospheric pressure chemical ionization mass spectrometry. Chin J Chromatogr 25:528-531

Huang XY, Fu JF, Di DL (2010) Preparative isolation and purification of steviol glycosides from Stevia rebaudiana Bertoni using high-speed counter-current chromatography. Sep Purif Technol 71:220-224

Huang J, Xu X, Xie C et al (2013) Isolation and purification of paeoniflorin and albiflorin from radix Paeoniae rubra by high-speed counter-current chromatography. J Liq Chromatogr Relat Technol 36:419-427

Ikai Y, Oka H, Hayakawa J et al (1998) Isolation of colistin C and B using high-speed countercurrent chromatography. J Liq Chromatogr Relat Technol 21:143-155

Ito Y (2005) Golden rules and pitfalls in selecting optimum conditions for high-speed counter-current chromatography. J Chromatogr A 1065:145-168

Ito Y, Weinstein M, Aoki I et al (1966) The coil planet centrifuge. Nature 212:985-987

Ito Y, Oka H, Lee YW (1990) Improved high speed counter current chromatograph with three multilayer coils connected in series. II. Separation of various biological samples with a semipreparative column. J Chromatogr 498: 169-178

Ito Y, Goto T, Yamada S et al (2006) Application of dual counter-current chromatography for rapid sample preparation of $\mathrm{N}$-methylcarbamate pesticides in vegetable oil and citrus fruit. J Chromatogr A 1108:20-25

Jiang Y, Lu HT, Chen F (2004) Preparative purification of glycyrrhizin extracted from the root of liquorice using high-speed counter-current chromatography. J Chromatogr A 1033:183-186

Jiang Z, Chen W, Liu S et al (2011) Preparation of crocin from Gardenia yellow pigment by slow rotary countercurrent chromatography. Chin J Chromatogr 29:277-280

Kim SM, Shang YF, Um BH (2011) A preparative method for isolation of fucoxanthin from Eisenia bicyclis by centrifugal partition chromatography. Phytochem Anal 22: 322-329 
Knapp H, Straubinger M, Fornari S et al (1998) \{S\}-3,7-dimethyl-5-octene-1,7-diol and related oxygenated monoterpenoids from petals of Rosa damascena Mill. J Agric Food Chem 46:1966-1970

Koch A, Basar S, Richter R (2008) TLC of mono- and sesquiterpenes. In: Waksmundzka-Hajnos M, Sherma J, Kowalska T (eds) Chromatography science series, vol 99. CRC Press, Boca Raton, pp 451-480

Lechtenberg M, Schepmann D, Niehues M et al (2008) Quality and functionality of saffron: quality control, species assortment and affinity of extract and isolated saffron compounds to NMDA and $\sigma_{1}$ (Sigma-1) receptors. Planta Med 74:764-772

Lee JH, Ha YW, Jeong CS et al (2009) Isolation and tandem mass fragmentations of an anti-inflammatory compound from Aralia elata. Arch Pharmacal Res 32:831-840

Li HB, Chen F (2001a) Preparative isolation and purification of astaxanthin from the microalga Chlorococcum sp. by highspeed counter-current chromatography. J Chromatogr 925:133-137

Li HB, Chen F (2001b) Preparative isolation and purification of six diterpenoids from the Chinese medicinal plant Salvia miltiorrhiza by high-speed counter-current chromatography. J Chromatogr A 925:109-114

Li HB, Chen F, Zhang TY et al (2001) Preparative isolation and purification of lutein from the microalga Chlorella vulgaris by high-speed counter-current chromatography. J Chromatogr 905:151-155

Li A, Sun A, Liu R (2005) Preparative isolation and purification of costunolide and dehydrocostuslactone from Aucklandia lappa Decne by high-speed counter-current chromatography. J Chromatogr A 1076:193-197

Li HB, Fan KW, Chen F (2006) Isolation and purification of canthaxanthin from the microalga Chlorella zofingiensis by high-speed counter-current chromatography. J Sep Sci 29:699-703

Li XC, Zhang QW, Yin ZQ et al (2011) Preparative separation of patchouli alcohol from patchouli oil using high performance centrifugal partition chromatography. J Essent Oil Res 23:19-24

Liang J, Meng J, Guo M et al (2013) Conical coils countercurrent chromatography for preparative isolation and purification of tanshinones from Salvia miltiorrhiza Bunge. J Chromatogr A 1288:35-39

Liu S, Cao M, Li D et al (2011) Purification and anticancer activity investigation of pentacyclic triterpenoids from the leaves of Sinojackia sarcocarpa L.Q. Luo by high-speed counter-current chromatography. Nat Prod Res 25:16001606

Liu J, Liu R, Sun A et al (2013a) Connection of high-speed counter-current chromatography with evaporative light scattering detector by flow injection and its application for preparative isolation and purification of ginkgolide compounds from Ginkgo biloba L. J Liq Chromatogr Relat Technol 36:2317-2329

Liu M, Yang F, Shi H et al (2013b) Preparative separation of triterpene alcohol ferulates from rice bran oil using a high performance counter-current chromatography. Food Chem 139:919-924

Lu HT, Jiang Y, Chen F (2003) Preparative separation and purification of squalene from the microalga
Thraustochytrium ATCC 26185 by high speed counter current chromatography. J Chromatogr 994:37-43

Lu Y, Sun C, Pan Y (2006) Isolation and purification of oridonin from Rabdosia rubescens using upright counter-current chromatography. J Sep Sci 29:314-318

Lu Y, Sun C, Liu R, Pan Y (2007) Effective two-dimensional counter-current chromatographic method for simultaneous isolation and purification of oridonin and ponicidin from the crude extract of Rabdosia rubescens. J Chromatogr A 1146:125-130

Ma Y, Aisha HA, Liao L et al (2005) Preparative isolation and purification of rupestonic acid from the Chinese medicinal plant Artemisia rupestris L. by high-speed counter-current chromatography. J Chromatogr A 1076:198-201

Ma J, Chen Q, Lai D et al (2010) Separation and purification of triterpene saponins from roots of Radix Phytolaccae by high-speed countercurrent chromatography coupled with evaporative light scattering detection. J Liq Chromatogr Relat Technol 33:563-571

Marston A, Borel C, Hostettmann K (1988) Separation of natural products by centrifugal partition chromatography. J Chromatogr 450:91-99

Martin AM, Queiroz EF, Marston A et al (2006) Labdane diterpenes from Juniperus communis L. berries. Phytochem Anal 17:32-35

Maurya A, Srivastava SK (2011) Preparative-scale separation of anticancer triterpenes from Eucalyptus hybrid by centrifugal partition chromatography. Sep Sci Technol 46: 1189-1194

Medhi B, Patyar S, Rao RS et al (2009) Pharmacokinetic and toxicological profile of artemisinin compounds: an update. Pharmacology 84:323-332

Mudge E, Lopes-Lutz D, Brown PN et al (2013) Purification of phenylalkanoids and monoterpene glycosides from Rhodiola rosea L. roots by high-speed counter-current chromatography. Phytochem Anal 24:129-134

Nasser ALM, Mazzolin LP, Hiruma-Lima CA et al (2006) Preparative droplet counter-current chromatography for the separation of the new nor-seco-triterpene and pentacyclic triterpenoids from Qualea parviflora. Chromatographia 64:695-699

Oka F, Oka H, Ito Y (1991) Systematic search for suitable twophase solvent systems for high speed counter-current chromatography. J Chromatogr 538:99-108

Peng A, Li R, Hu J et al (2008a) Flow rate gradient high-speed counter-current chromatography separation of five diterpenoids from Triperygium wilfordii and scale-up. J Chromatogr A 1200:129-135

Peng J, Dong F, Qi Y et al (2008b) Preparative separation of four triterpene saponins from Radix Astragali by highspeed counter-current chromatographycoupled with evaporative light scattering detection. Phytochem Anal 19:212-217

Pinel B, Audo G, Mallet S et al (2007) Multi-grams scale purification of xanthanolides from Xanthium macrocarpum. Centrifugal partition chromatography versus silica gel chromatography. J Chromatogr A 1151:14-19

Puertas Mejia M, Hillebrand S, Stashenko E et al (2002) In vitro radical scavenging activity of essential oils from Columbian plants and fractions from oregano (Origanum vulgare L) essential oil. Flavour Fragr J 17:380-384 
Qi X, Ignatova S, Luo G et al (2010) Preparative isolation and purification of ginsenosides Rf, Re, Rd and Rb1 from the roots of Panax ginseng with a salt/containing solvent system and flow step-gradient by high performance countercurrent chromatography coupled with an evaporative light scattering detector. J Chromatogr A 1217:1995-2001

Qiu F, Friesen J, McAlpinea JB et al (2012) Design of countercurrent separation of Ginkgo biloba terpene lactones by nuclear magnetic resonance. J Chromatogr A 1242:26-34

Rocha GG, Simões M, Lúcio KA et al (2007) Natural triterpenoids from Cecropia lyratiloba are cytotoxic to both sensitive and multidrug resistant leukemia cell lines. Bioorgan Med Chem 15:7355-7360

Rodrigues VF, Carmo HM, Oliveira RR et al (2009) Isolation of terpenoids from Trichilia quadrijuga (Meliaceae) by droplet counter-current chromatography. Chromatographia 70:1191-1195

Roscher R, Winterhalter P (1993) 1,1,6-trimethyl-1,2-dihydronaphthalene (TDN) formation in wine. Application of multilayer coil countercurrent chromatography for the study of Vitis vinifera cv. Riesling leaf glycosides. J Agric Food Chem 41:1452-1457

Severino VGP, Cazal CM, Forim MR et al (2009) Isolation of secondary metabolites from Hortia oreadica (Rutaceae) leaves through high-speed counter-current chromatography. J Chromatogr A 1216:4275-4281

Shehzad O, Jin Ha I et al (2011) Development of a rapid and convenient method to separate eight ginsenosides from Panax ginseng by high-speed counter-current chromatography coupled with evaporative light scattering detection. J Sep Sci 34:1116-1122

Shehzad O, Khan S, Ha IJ et al (2012) Rational development of a selection model for solvent gradients in single-step separation of ginsenosides from Panax ginseng using highspeed counter-current chromatography. J Sep Sci 35:1462-1469

Shehzad O, Kim HP, Kim YS (2013) State-of-the-art separation of ginsenosides from Korean white and red ginseng by countercurrent chromatography. Anal Bioanal Chem 405:4523-4530

Shi S, Jiang D, Zhao M et al (2007) Preparative isolation and purification of triterpene saponins from Clematis mandshurica by high-speed counter-current chromatography coupled with evaporative light scattering detection. J Chromatogr B 852:679-683

Shi SY, Zhou HH, Huang KL et al (2008) Application of highspeed counter-current chromatography for the isolation of antiviral eremophilenolides from Ligularia atroviolacea. Biomed Chromatogr 22:985-991

Shi X, Wang X, Wang D et al (2009) Separation and purification of $\alpha$-cyperone from Cyperus rotundus with supercritical fluid extraction and high-speed counter-current chromatography. Sep Sci Technol 44:712-721

Shirota O, Nagamatsu K, Sekita S (2007) Simple preparative isolation of salvinorin a from the hallucinogenic sage, Salvia divinorum, by centrifugal partition chromatography. J Liq Chromatogr Relat Technol 30:1105-1114

Shirota O, Nagamatsu K, Sekita S et al (2008) Preparative separation of the saponin lancemaside A from Codonopsis lanceolata by centrifugal partition chromatography. Phytochem Anal 19:403-410
Silva JCT, Jham GN, Oliveira RDL et al (2007) Purification of the seven tetranortriterpenoids in neem (Azadirachta indica) seed by counter-current chromatography sequentially followed by isocratic preparative reversed-phase high-performance liquid chromatography. J Chromatogr A 1151:203-210

Skalicka-Woźniak K, Walasek M, Ludwiczuk A et al (2013) Isolation of terpenoids from Pimpinella anisum essential oil by high-performance counter-current chromatography. J Sep Sci 36:2611-2614

Slacanin I, Marston A, Hostettmann K et al (1991) The isolation of Eleutherococcus senticosus constituents by centrifugal partition chromatography and their quantitative determination by high performance liquid chromatography. Phytochem Anal 2:137-142

Sun A, Zhang Y, Li A et al (2011) Extraction and preparative purification of tanshinones from Salvia miltiorrhiza Bunge by high-speed counter-current chromatography. J Chromatogr B Anal Technol Biomed Life Sci 879:1899-1904

Sutherland IA, Fisher D (2004) Dynamic extraction technology. Innov Pharm Technol 10:68-71

Sutherland IA, Fisher D (2009) Role of counter-current chromatography in the modernisation of Chinese herbal medicines. J Chromatogr A 1216:740-753

Sutherland IA, Brown L, Forbes S et al (1998) Countercurrent chromatography (CCC) and its versatile application as an industrial purification and production process. J Liq Chromatogr Relat Technol 21:279-298

Tian G, Zhang Y, Zhang T et al (2000) Separation of tanshinones from Salvia miltiorrbiza Bunge by high-speed counter-current chromatography. J Chromatogr 904:107-111

Tian G, Zhang T, Zhang Y et al (2002) Separation of tanshinones from Salvia miltiorrhiza Bunge by multidimensional counter current chromatography. J Chromatogr A 945:281-285

Tsao R, Yang R (2006) Lutein in selected Canadian crops and agri-food processing by-products and purification by highspeed counter-current chromatography. J Chromatogr A 1112:202-208

Van den Heuvel R (2007) Investigation into the mechanics and feasibility of continuous counter-current extraction. $\mathrm{PhD}$ Thesis, Brunel Univeristy, London, UK

Wang G, Tang W, Bidigare RR (2005) Terpenoids as therapeutic drugs and pharmaceutical agents. In: Zhang L, Demain AL (eds) Natural products: drug discovery and therapeutic medicine. Humana Press Inc., Totowa, pp 197-227

Wang J, Bai HL, Liu CM et al (2010) Isolation and purification of ginsenosides from plant extract of Panax quinquefolium $L$. by high performance centrifugal partition chromatography coupled with ELSD. Chromatographia 71:267-271

Wang D, Fang L, Wang X et al (2011a) Preparative separation and purification of sesquiterpenoids from Tussilago farfara L. by high-speed counter-current chromatography. Quim Nova 34:804-807

Wang J, Liu CM, Li L et al (2011b) Isolation of four high-purity dammarane saponins from extract of Panax notoginseng by centrifugal partition chromatography coupled with evaporative light scattering detection in one operations. Phytochem Anal 22:263-267 
Wang D, Bai A, Lin X et al (2012a) Efficient method for extraction and isolation of shionone from Aster tataricus $\mathrm{L}$. f. by supercritical fluid extraction and high-speed countercurrent chromatography. Acta Chromatogr 24:615-625

Wang Y, Liu M, Zheng L et al (2012b) Preparative purification of five bioactive components from Agrimonia pilosa Ledeb by high-speed counter-current chromatography. J Sep Sci 35:1977-1984

Wang J, Yu J, Li L et al (2013) Isolation of high purity ginsenosides from plant extract of Panax ginseng by high performance centrifugal partition chromatography coupled with evaporative light scattering detection. J Liq Chromatogr Relat Technol 36:583-590

Wei Y, Zhang T, Xu G et al (2001) Application of analytical and preparative high-speed counter-current chromatography for separation of lycopene from crude extract of tomato paste. J Chromatogr A 929:169-173

Wei Y, Zhang T, Xu G et al (2003) Application of CCC for the separation of lutein from a crude extract of marigold flower petals. J Liq Chromatogr Relat Technol 26:1659-1669

Wei Y, Du J, Lu Y (2012) Preparative separation of bioactive compounds from essential oil of Flaveria bidentis (L.) Kuntze using steam distillation extraction and one step high-speed counter-current chromatography. J Sep Sci 35:2608-2614

Wu S, Sun C, Wang K et al (2004) Preparative isolation and purification of celastrol from Celastrus orbicularis Thunb. by a new counter current chromatography method with an upright coil planet centrifuge. J Chromatogr A 1028: 171-174

Wu H, Su Z, Yang Y et al (2007) Isolation of three sesquiterpene lactones from the roots of Cichorium glandulosum Boiss. et Huet. by high-speed counter-current chromatography. J Chromatogr A 1176:217-222

Wu S, Yang L, Gao Y et al (2008) Multi-channel counter-current chromatography for high-throughput fractionation of natural products for drug discovery. J Chromatogr A 1180:99-107

Wu D, Jiang X, Wu S (2010) Direct purification of tanshinones from Salvia miltiorrhiza Bunge by high-speed countercurrent chromatography without presaturation of the twophase solvent mixture. J Sep Sci 33:67-73

Xiang F, Yu J, Yin R et al (2009) Structure-activity relationship of Taxol inferring from docking Taxol analogues to microtubule binding site. Z Naturforsch $64 \mathrm{c}$ : 551-556

Xiao X, Si X, Yuan Z et al (2012) Isolation of fucoxanthin from edible brown algae by microwave-assisted extraction coupled with high-speed countercurrent chromatography. J Sep Sci 35:2313-2317

Xiao XH, Yuan ZQ, Li GK (2013) Preparation of phytosterols and phytol from edible marine algae by microwave-assisted extraction and high-speed counter-current chromatography. Sep Purif Technol 104:284-289

Xie J, Wang S, Sun B et al (2008) Preparative separation and purification of $\beta$-caryophyllene from leaf oil of Vitex negundo L. var. heterophylla (Franch.) Rehd. by high speed countercurrent chromatography. J Liq Chromatogr Relat Technol 31:2621-2631

Xie J, Sun B, Wang S et al (2009) Isolation and purification of nootkatone from the essential oil of fruits of Alpinia oxyphylla Miquel by high-speed counter-current chromatography. Food Chem 117:375-380

Xin X, Yang Y, Zhong J et al (2009) Preparative isolation and purification of isobenzofuranone derivatives and saponins from seeds of Nigella glandulifera Freyn by high-speed counter-current chromatography combined with gel filtration. J Chromatogr A 1216:4258-4262

Xu J, Luo J, Kong L (2013) Simultaneous separation of triterpenoid saponins and flavonoid glycosides from the roots of Glycyrrhiza uralensis Fisch by $\mathrm{pH}$-zone-refining countercurrent chromatography. J Sep Sci 36:3295-3301

Yan J, Chen G, Tong S et al (2005) Preparative isolation and purification of germacrone and curdione from the essential oil of the rhizomes of Curcuma wenyujin by high-speed counter-current chromatography. J Chromatogr A 1070: 207-210

Yan G, Ji L, Luo Y et al (2012) Preparative isolation and purification of three sesquiterpenoid lactones from Eupatorium lindleyanum DC. by high-speed counter-current chromatography. Molecules 17:9002-9009

Yang F, Ou Q, Yu W (1995) Semi-preparative separation of taraxeryl-acetate and coumarins from Artemesia dalailamae Kraschen by high-speed countercurrent chromatography. J Liq Chromatogr 18:395-403

Yang Y, Gu D, Yili A et al (2010) One-step separation and purification of rupestonic acid and chrysosptertin B from Artemisia rupestris $L$. by high-speed counter-current chromatography. Phytochem Anal 21:205-209

Yang Y, Bakri M, Gu D et al (2013) Separation of (S)-dehydrovomifoliol from leaves of Nitraria sibirica Pall. by high-speed counter-current chromatography. J Liq Chromatogr Relat Technol 36:573-582

Yao S, Liu R, Huang X et al (2007) Preparative isolation and purification of chemical constituents from the root of $\mathrm{Ad}$ enophora tetraphlla by high-speed counter-current chromatography with evaporative light scattering detection. J Chromatogr A 1139:254-262

Yao S, Luo J, Huang X et al (2008) Application of preparative high-speed counter-current chromatography/preparative high-performance liquid chromatography mode in rapid separation of saponins. J Chromatogr B 864:69-77

Ye H, Chen L, Li Y et al (2008a) Preparative isolation and purification of three rotenoids and one isoflavone from the seeds of Millettia pachycarpa Benth by high-speed counter-current chromatography. J Chromatogr A 1178: 101-107

Ye H, Ignatova S, Luo H et al (2008b) Preparative separation of a terpenoid and alkaloids from Tripterygium wilfordii Hook. using high-performance counter-current chromatography. Comparison of various elution and operating strategies. J Chromatogr A 1213:145-153

Yoo KY, Park SY (2012) Terpenoids as potential anti-Alzheimer's disease therapeutics. Molecules 17:3524-3538

Yoon KD, Kim J (2009) Application of centrifugal partition chromatography coupled with evaporative light scattering detection for the isolation of saikosaponins-a and -c from Bupleurum falcatum roots. J Sep Sci 32:74-78

Yoon KD, Chin YW, Ahn MJ et al (2009) Preparative isolation of alisol B and alisol b 23-acetate from Alismatis rhizoma by centrifugal partition chromatography coupled with ELSD. Chromatographia 69:791-793 
Yuan Y, Wang B, Chen L et al (2008) How to realize the linear scale-up process for rapid purification using high-performance counter-current chromatography. J Chromatogr A 1194:192-198

Zhang H, Feng B, Liu K et al (2013a) Isolation and purification of two triterpenoids from the Chinese medicinal plant Fomes officinalis Ames. Asian J Chem 25:6130-6132

Zhang Y, Liu C, Qi Y et al (2013b) Application of accelerated solvent extraction coupled with counter-current chromatography to extraction and online isolation of saponins with a broad range of polarity from Panax notoginseng. Sep Purif Technol 106:82-89
Zhao Y, Du Q (2007) Separation of solanesol in tobacco leaves extract by slow rotary counter-current chromatography using a novel non-aqueous two-phase solvent system. J Chromatogr A 1151:193-196

Zhao C, He C (2006) Preparative isolation and purification of atractylon and atractylenolide III from the Chinese medicinal plant Atractylodes macrocephala by high-speed counter-current chromatography. J Sep Sci 29:1630-1636

Zhao D, Yan M, Huang Y et al (2012) Efficient protocol for isolation and purification of different soyasaponins from soy hypocotyls. J Sep Sci 35:3281-3292 